\title{
Sterile neutrino oscillations after first MiniBooNE results
}

\author{
Michele Maltoni ${ }^{1, *}$ and Thomas Schwetz ${ }^{2, \dagger}$ \\ ${ }^{1}$ Departamento de Física Teórica and Instituto de Física Teórica UAM/CSIC, Facultad de Ciencias C-XI, \\ Universidad Autónoma de Madrid, Cantoblanco, E-28049 Madrid, Spain \\ ${ }^{2}$ Physics Department, Theory Division, CERN, CH-1211 Geneva 23, Switzerland
}

(Received 14 June 2007; published 19 November 2007)

\begin{abstract}
In view of the recent results from the MiniBooNE experiment we revisit the global neutrino oscillation fit to short-baseline neutrino data by adding one or two sterile neutrinos with eV-scale masses to the three standard model neutrinos, and for the first time we consider also the global fit with three sterile neutrinos. Four-neutrino oscillations of the $(3+1)$ type have been only marginally allowed before the recent MiniBooNE results, and become even more disfavored with the new data (at the level of $4 \sigma$ ). In the framework of so-called $(3+2)$ five-neutrino mass schemes we find severe tension between appearance and disappearance experiments at the level of more than $3 \sigma$, and hence no satisfactory fit to the global data is possible in $(3+2)$ schemes. This tension remains also when a third sterile neutrino is added, and the quality of the global fit does not improve significantly in a $(3+3)$ scheme. It should be noted, however, that in models with more than one sterile neutrino the MiniBooNE results are in perfect agreement with the appearance evidence reported by the LSND Collaboration, thanks to the possibility of $C P$ violation available in such oscillation schemes. Furthermore, if disappearance data are not taken into account $(3+$ 2) oscillations provide an excellent fit to the full MiniBooNE spectrum including the event excess at low energies.
\end{abstract}

DOI: 10.1103/PhysRevD.76.093005

PACS numbers: $14.60 . P q$

\section{INTRODUCTION}

Recently first results from the MiniBooNE (MB) experiment $[1,2]$ at Fermilab have been released on a search for $\nu_{\mu} \rightarrow \nu_{e}$ appearance with a baseline of $540 \mathrm{~m}$ and a mean neutrino energy of about $700 \mathrm{MeV}$. The primary purpose of this experiment is to test the evidence of $\bar{\nu}_{\mu} \rightarrow \bar{\nu}_{e}$ transitions found by the LSND experiment at Los Alamos [3] with a very similar $L / E$ range. Reconciling the LSND signal with the other evidence for neutrino oscillations is a long-standing challenge for neutrino phenomenology, since it requires a mass-squared difference at the $\mathrm{eV}^{2}$ scale, at odds with the values needed to explain atmospheric [4], long-baseline accelerator [5,6], solar [7-9], and longbaseline reactor [10] neutrino data.

It turns out that introducing a fourth (sterile) neutrino [11] does not lead to a satisfactory description of all data in terms of neutrino oscillations [12,13] because of tight constraints from atmospheric [4], solar [9], and null-result short-baseline (SBL) experiments [14-17] (see Ref. [18] for early four-neutrino analyses considering LSND, and Refs. [19,20] for recent updates). So-called $(2+2)$ schemes are ruled out by strong constraints on a sterile neutrino component in solar as well as in atmospheric neutrino oscillations [21] at high significance. Therefore, we will not consider such schemes in the following. Also $(3+1)$ schemes suffer from a well-known tension between the LSND appearance signal and null-result SBL disappearance experiments [22-28]. We will show that recent MB results further aggravate this tension and hence $(3+1)$

\footnotetext{
*maltoni@delta.ft.uam.es

${ }^{\dagger}$ schwetz@cern.ch
}

schemes get even more disfavored. In Ref. [29] a fiveneutrino mass scheme of the $(3+2)$ type has been considered, arguing that the disagreement between LSND and null-result experiments becomes somewhat relaxed compared to $(3+1)$; see also Ref. [26]. Here we reconsider this possibility in view of the recent MB data. Furthermore, we investigate the impact of adding a third sterile neutrino on the quality of the global fit. Since we know that there are three active neutrinos, the possibility of three sterile neutrinos is appealing for aesthetical reasons.

Apart from sterile neutrino oscillations, various more exotic explanations of the LSND signal have been proposed, for example, neutrino decay [30,31], $C P T$ violation [13,32], violation of Lorentz symmetry [33], a lepton number violating muon decay [34], $C P T$-violating quantum decoherence [35], mass-varying neutrinos [36], or shortcuts of sterile neutrinos in extra dimensions [37].

In this work we concentrate on the oscillation framework including one or more sterile neutrinos at the $\mathrm{eV}$ scale. Such models do have an impact on cosmology [38]. First, the sterile neutrinos will contribute to the effective number of neutrino species at big bang nucleosynthesis $[23,39]$, and second, these models are subject to strong bounds on the sum of the neutrino masses in the sub-eV range from the combination of various cosmological data sets (see, e.g., Ref. [40] for recent analyses). In order to reconcile such neutrino schemes with cosmology some nonstandard scenario has to be invoked; see for example Refs. [41-43].

The outline of the paper is as follows. In Sec. II we consider $(3+1)$ four-neutrino schemes, and give some details on the used data and their analysis. Section III is devoted to $(3+2)$ five-neutrino schemes, discussing the 
compatibility of LSND and MB in such schemes, as well as the problems of these models to reconcile appearance and disappearance experiments. In Sec. IV we extend the $(3+$ 2) scheme by adding a third sterile neutrino to a $(3+3)$ six-neutrino model and investigate whether the global fit improves significantly. We summarize in Sec. V. In Appendix A we discuss the mechanism to reconcile LSND and MB by $C P$ violation in $(3+2)$ schemes, Appendix B contains a parameter counting of general sterile neutrino oscillation schemes, and in Appendix C we consider details of the analysis of atmospheric neutrino data in such models.

\section{II. $(3+1)$ FOUR-NEUTRINO MASS SCHEMES}

The $(3+1)$ four-neutrino spectra are a small perturbation to the standard three-active neutrino case. A cluster of three neutrino mass states accounts for the "solar" $\left(\Delta m_{21}^{2}\right)$ and "atmospheric" $\left(\Delta m_{31}^{2}\right)$ mass splittings. The fourth mass state is separated by an eV-scale mass gap to account for the LSND oscillations, and there is only small mixing of active neutrinos with this mass eigenstate.

\section{A. Appearance data in $(3+1)$ schemes}

We start our discussion of the $(3+1)$ mass scheme by considering the SBL appearance experiments in the $\nu_{\mu} \rightarrow$ $\nu_{e}\left(\right.$ or $\bar{\nu}_{\mu} \rightarrow \bar{\nu}_{e}$ ) channel, including the LSND [3] evidence, the bounds from KARMEN [14] and NOMAD [15], and the recent MB [1] data. A combined analysis of LSND and KARMEN can be found in Ref. [44]. In the approximation $\Delta m_{21}^{2} \approx \Delta m_{31}^{2} \approx 0$, the SBL appearance probability in $(3+1)$ schemes is equivalent to the twoneutrino case, where the effective mixing angle is determined by $\sin ^{2} 2 \theta_{\mathrm{SBL}}=4\left|U_{e 4}\right|^{2}\left|U_{\mu 4}\right|^{2}$. Therefore, the analysis performed by the MB Collaboration [1,2] directly applies to $(3+1)$ schemes. We comment only briefly on this case, with the main purpose to check our analysis against the official MB results.

For our reanalysis of LSND we fit the observed transition probability (total rate) plus 11 data points of the $L / E$ spectrum with free normalization, both derived from the decay-at-rest data [3]. For KARMEN the data observed in 9 bins of prompt energy as well as the expected background [14] is used in the fit. Further details of our LSND and KARMEN analyses are given in Ref. [31]. For NOMAD we fit the total rate using the information provided in Ref. [15]; our exclusion curve is in good agreement with the result presented in that reference.

The MB reanalysis is based on the $\nu_{\mu}$ neutrino flux, efficiencies, and energy resolution provided in Ref. [2], folded with the $\nu_{e}$ charged-current quasielastic (CCQE) cross section, to obtain a prediction for the CCQE event excess from $\nu_{\mu} \rightarrow \nu_{e}$ oscillations. We calibrate our simulation to the official $\mathrm{MB}$ analysis using the prediction for two example points provided in Ref. [1]. For the fit the spectrum of excess events binned in reconstructed neutrino energy from Fig. 2 of Ref. [1] is used, where the error bars include statistical errors and the uncertainty from the background prediction. Detailed technical information on the MB oscillation analysis is available in Ref. [45], including efficiencies and error correlations. Our MB results are in good agreement with the official MB analysis as described in Ref. [45].

MB data are consistent with zero (no excess) above $475 \mathrm{MeV}$, whereas below this energy a $3.6 \sigma$ excess of $96 \pm$ $17 \pm 20$ events is observed. Whether this excess comes indeed from $\nu_{\mu} \rightarrow \nu_{e}$ transitions or has some other origin is under investigation [1]. Lacking any explanation in terms of backgrounds or systematical uncertainties we take these data at face value, and in some cases we will use all 10 bins of the full energy range from $300 \mathrm{MeV}$ to $3 \mathrm{GeV}$ in the fit ("MB300"). However, as discussed in Refs. [1,2], two-neutrino oscillations cannot account for the event excess at low energies. We confirm that the quality of the $(3+1) \mathrm{MB}$ fit drastically worsens when the two energy bins between 300 and $475 \mathrm{MeV}$ are included in the fit. Therefore, we follow the strategy of the MB Collaboration and restrict the $(3+1)$ analysis to the energy range from $475 \mathrm{MeV}$ to $3 \mathrm{GeV}$ ("MB475").

The bound from MB475 data is shown in Fig. 1 in comparison with the allowed region from the combined LSND, KARMEN, NOMAD data. In agreement with Refs. [1,2] we find that the $90 \%$ C.L. regions do not overlap. A marginal overlap appears if both data sets are stretched to the $99 \%$ C.L. If all data are summed we find a best fit point with $\chi_{\min }^{2}=26.6$ for $(29-2)$ degrees of freedom (dof). Although this leads to a very good nominal

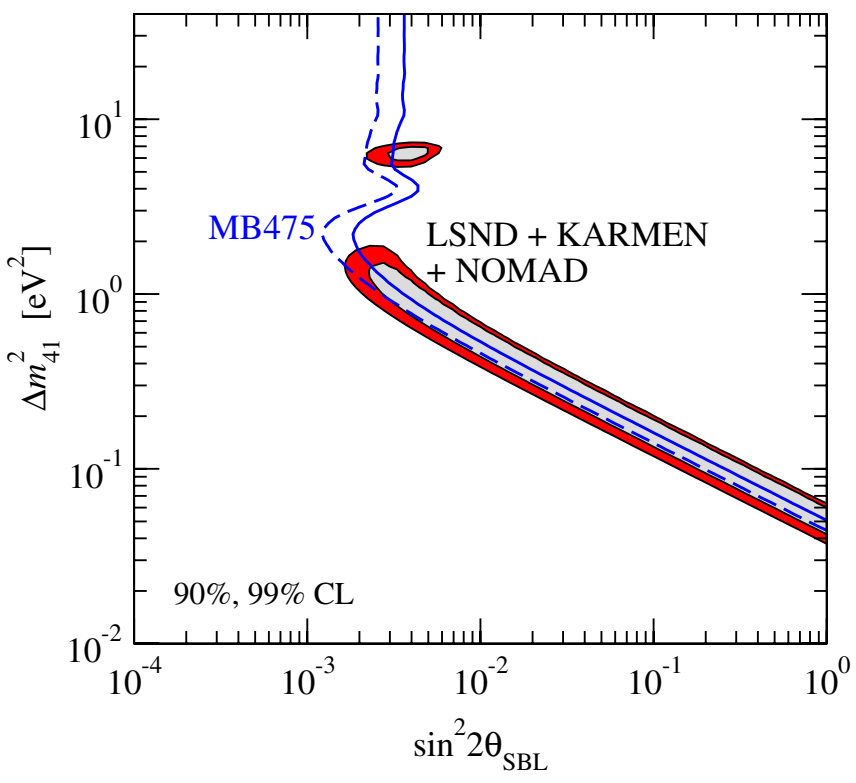

FIG. 1 (color online). Allowed region for MB475 (solid and dashed curves) and LSND + KARMEN + NOMAD (shaded regions) at $90 \%$ and $99 \%$ C.L. $(2$ dof $)$ in $(3+1)$ mass schemes. 
goodness-of-fit (gof), the figure clearly shows that there is significant tension between MB and LSND. A powerful tool to evaluate the compatibility of different data sets is the so-called parameter goodness-of-fit (PG) criterion discussed in Ref. [46]. It is based on the $\chi^{2}$ function

$$
\chi_{\mathrm{PG}}^{2}=\chi_{\mathrm{tot}, \min }^{2}-\sum_{i} \chi_{i, \min }^{2}
$$

where $\chi_{\text {tot,min }}^{2}$ is the $\chi^{2}$ minimum of all data sets combined and $\chi_{i, \min }^{2}$ is the minimum of the data set $i$. This $\chi^{2}$ function measures the "price" one has to pay by the combination of the data sets compared to fitting them independently. It should be evaluated for the number of dof corresponding to the number of parameters in common to the data sets; see Ref. [46] for a precise definition. Applying this test to check the compatibility of MB with the other SBL appearance data, we find $\chi_{\mathrm{PG}}^{2}=7.4$ ( 2 dof), corresponding to a PG of $2.5 \%$. If we test the compatibility of the three data sets MB, LSND, and KARMEN + NOMAD we find $\chi_{\mathrm{PG}}^{2}=13.7$ (4 dof), and $\mathrm{PG}=0.8 \%$. In the latter case the slight tension between LSND and KARMEN also contributes to the $\chi^{2}$, whereas in the first case this tension is removed since they are added into one single data set.

\section{B. Global SBL data in $(3+1)$ schemes}

Now we proceed to the global four-neutrino analysis, adding also the information from disappearance experiments. We include the Bugey [17], Chooz [47], and Palo Verde [48] $\bar{\nu}_{e}$ reactor experiments, as well as the CDHS [16] $\nu_{\mu}$ disappearance experiment. Details of our Bugey and CDHS fits can be found in Ref. [27]. Furthermore, atmospheric neutrino data give an important constraint on the parameter $d_{\mu}$ [25,28], which is equal to $\left|U_{\mu 4}\right|^{2}$ in $(3+1)$ schemes; see Ref. [49] and appendices B and $\mathrm{C}$. This information is crucial for the region of $\Delta m_{41}^{2} \lesssim$ $1 \mathrm{eV}^{2}$, where the bound from CDHS disappears. We use the updated atmospheric neutrino analysis from Ref. [20], which includes also recent K2K [5] and MINOS [6] data, and include the bound on $d_{\mu}$ as one single data point in the SBL fit. The total number of data points in the $(3+1)$ analysis is

$$
\left.\begin{array}{c}
N_{\mathrm{APP}}=11_{(\mathrm{LSND})}+9_{(\mathrm{KARMEN})}+1_{(\mathrm{NOMAD})}+8_{(\mathrm{MB} 475)}=29 \\
N_{\mathrm{DIS}}=60_{(\text {Bugey })}+1_{(\mathrm{Chooz})}+1_{(\text {PaloVerde })}+15_{(\mathrm{CDHS})}+1_{(\mathrm{ATM})}=78
\end{array}\right\} \quad N_{\text {tot }}=107 .
$$

It is well known that $(3+1)$ schemes suffer from a tension between the LSND appearance signal and the bounds from disappearance experiments; see, e.g., Refs. [22-27]. Reactor experiments constrain the parameter $\left|U_{e 4}\right|^{2} \ll 1$, CDHS and atmospheric neutrinos limit $\left|U_{\mu 4}\right|^{2} \ll 1$, whereas the LSND oscillation amplitude is given by $4\left|U_{e 4}\right|^{2}\left|U_{\mu 4}\right|^{2}$. Because of this tension $(3+1)$ schemes have been already disfavored before the recent MB results; see Ref. [19]. Testing the compatibility of LSND with all the no-evidence experiments (NEV) without MB leads to a $\chi_{\mathrm{PG}}^{2}=20.9$ for 2 dof, which indicates an inconsistency at high C.L. Since MB475 data is also in conflict with the LSND signal the new data adds to the tension and we find

$$
\chi_{\mathrm{PG}}^{2}=24.7(2 \mathrm{dof}), \quad \text { LSND vs NEV(incl. MB475). }
$$

Alternatively one may test the compatibility of the three data sets LSND, MB475 + KARMEN + NOMAD (NEVAPP), and disappearance experiments, which gives

$$
\chi_{\mathrm{PG}}^{2}=24.8(4 \mathrm{dof}), \quad \text { LSND vs NEV-APP vs DIS. }
$$

These numbers formally imply inconsistency at more than $4 \sigma$, and hence we conclude that $(3+1)$ schemes are highly disfavored by recent data. This level of incompatibility is already close to the one of solar and atmospheric neutrino data in $(2+2)$ four-neutrino schemes $[12,19,20]$ with a
$\chi_{\mathrm{PG},(2+2)}^{2}=30.7$ (1 dof). Let us mention that the global best fit point of all data has $\chi_{\min }^{2}=100.7$ for $(107-$ 3 ) dof, which nominally gives an excellent gof. However, in the standard gof test the problems in the fit get diluted by the large number of data points, and the disagreement of different data sets becomes only visible when they are analyzed separately and then compared to each other; see Ref. [46] for a detailed discussion.

The bound from NEV data in comparison with the LSND region is shown in Fig. 2. Let us mention that the shape of the NEV bound does hardly change by adding MB data, however the $\chi^{2}$ of the global best fit point increases significantly (see above). Note also that for our LSND analysis we use only the decay-at-rest data, where the appearance signal is most significant. ${ }^{1}$ If the global LSND data including also the decay-in-flight events are used, the LSND regions shift to slightly smaller values of $\sin ^{2} 2 \theta_{\text {SBL }}$ and the disagreement with NEV gets somewhat less severe; see Ref. [12] for a discussion of this issue.

To summarize, $(3+1)$ schemes get further disfavored by MB data for two reasons. First, in these models LSND

\footnotetext{
${ }^{1}$ The LSND allowed region of Fig. 2 consists of a connected band, which shows that the fit is dominated by the total rate and the spectral information available to us is not strong enough to produce disconnected regions as obtained from an event-based likelihood analysis [44]. However, the location and size of our region are in very good agreement with Ref. [44], and, moreover, the regions of the parameter space which are relevant for the combination with NEV data are reproduced very well.
} 


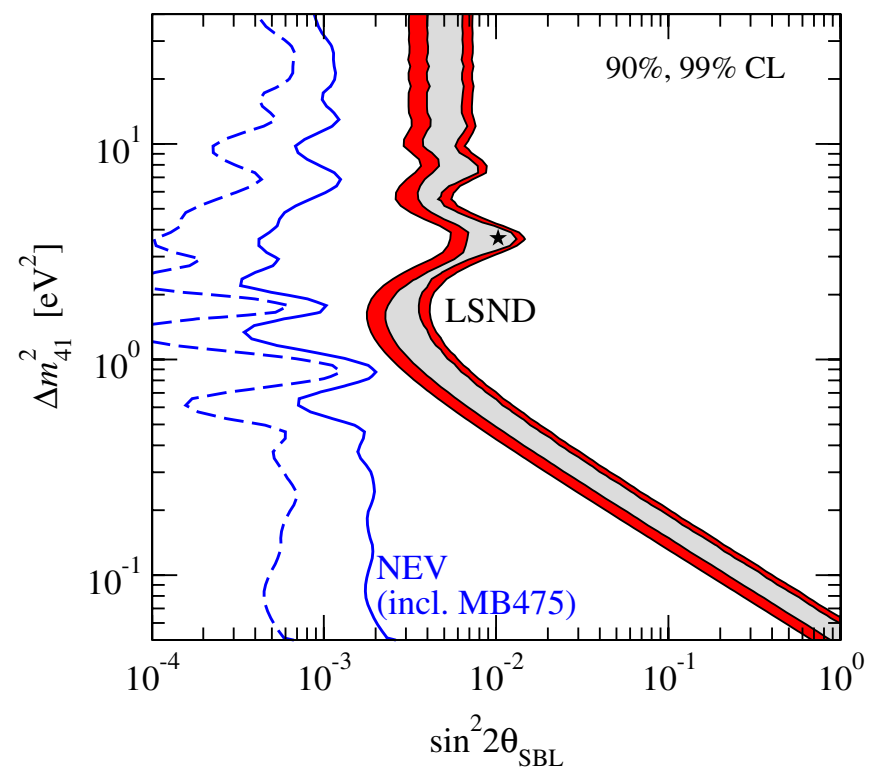

FIG. 2 (color online). Allowed regions in $(3+1)$ schemes from no-evidence (NEV) data including MB475 (solid and dashed curves) and LSND (shaded regions) at $90 \%$ and 99\% C.L. (2 dof).

and $\mathrm{MB}$ are in disagreement at the level of $90 \%$ to $98.5 \%$ C.L. $[1,2]$, and second, the tension between LSND and $\mathrm{NEV}$ data gets worse due to $\mathrm{MB}$.

\section{III. (3 + 2) FIVE-NEUTRINO MASS SCHEMES}

Five-neutrino schemes of the $(3+2)$ type are a straightforward extension of $(3+1)$ schemes. In addition to the cluster of the three neutrino mass states accounting for solar and atmospheric mass splittings now two states at the $\mathrm{eV}$ scale are added, with a small admixture of $\nu_{e}$ and $\nu_{\mu}$ to account for the LSND signal. In Ref. [29] it has been argued that in $(3+2)$ schemes the tension between LSND and NEV data becomes significantly relaxed compared to the $(3+1)$ case. Here we reconsider this possibility in the light of the new MB data.

\section{A. Appearance data in $(3+2)$ schemes}

First we consider appearance data only (LSND, KARMEN, NOMAD, and MB). In the SBL approximation $\Delta m_{21}^{2} \approx \Delta m_{31}^{2} \approx 0$, the relevant appearance probability is given by

$$
\begin{aligned}
P_{\nu_{\mu} \rightarrow \nu_{e}}= & 4\left|U_{e 4}\right|^{2}\left|U_{\mu 4}\right|^{2} \sin ^{2} \phi_{41}+4\left|U_{e 5}\right|^{2}\left|U_{\mu 5}\right|^{2} \sin ^{2} \phi_{51} \\
& +8\left|U_{e 4} U_{\mu 4} U_{e 5} U_{\mu 5}\right| \sin \phi_{41} \sin \phi_{51} \cos \left(\phi_{54}-\delta\right),
\end{aligned}
$$

with the definitions

$$
\phi_{i j} \equiv \frac{\Delta m_{i j}^{2} L}{4 E}, \quad \delta \equiv \arg \left(U_{e 4}^{*} U_{\mu 4} U_{e 5} U_{\mu 5}^{*}\right)
$$

Equation (5) holds for neutrinos (NOMAD and MB); for antineutrinos (LSND and KARMEN) one has to replace $\delta \rightarrow-\delta$. Since Eq. (5) is invariant under the transformation $4 \leftrightarrow 5$ and $\delta \rightarrow-\delta$, we can restrict the parameter range to $\Delta m_{54}^{2} \geq 0$, or equivalently $\Delta m_{51}^{2} \geq \Delta m_{41}^{2}$, without loss of generality. Note also that the probability Eq. (5) depends only on the combinations $\left|U_{e 4} U_{\mu 4}\right|$ and $\left|U_{e 5} U_{\mu 5}\right|$,

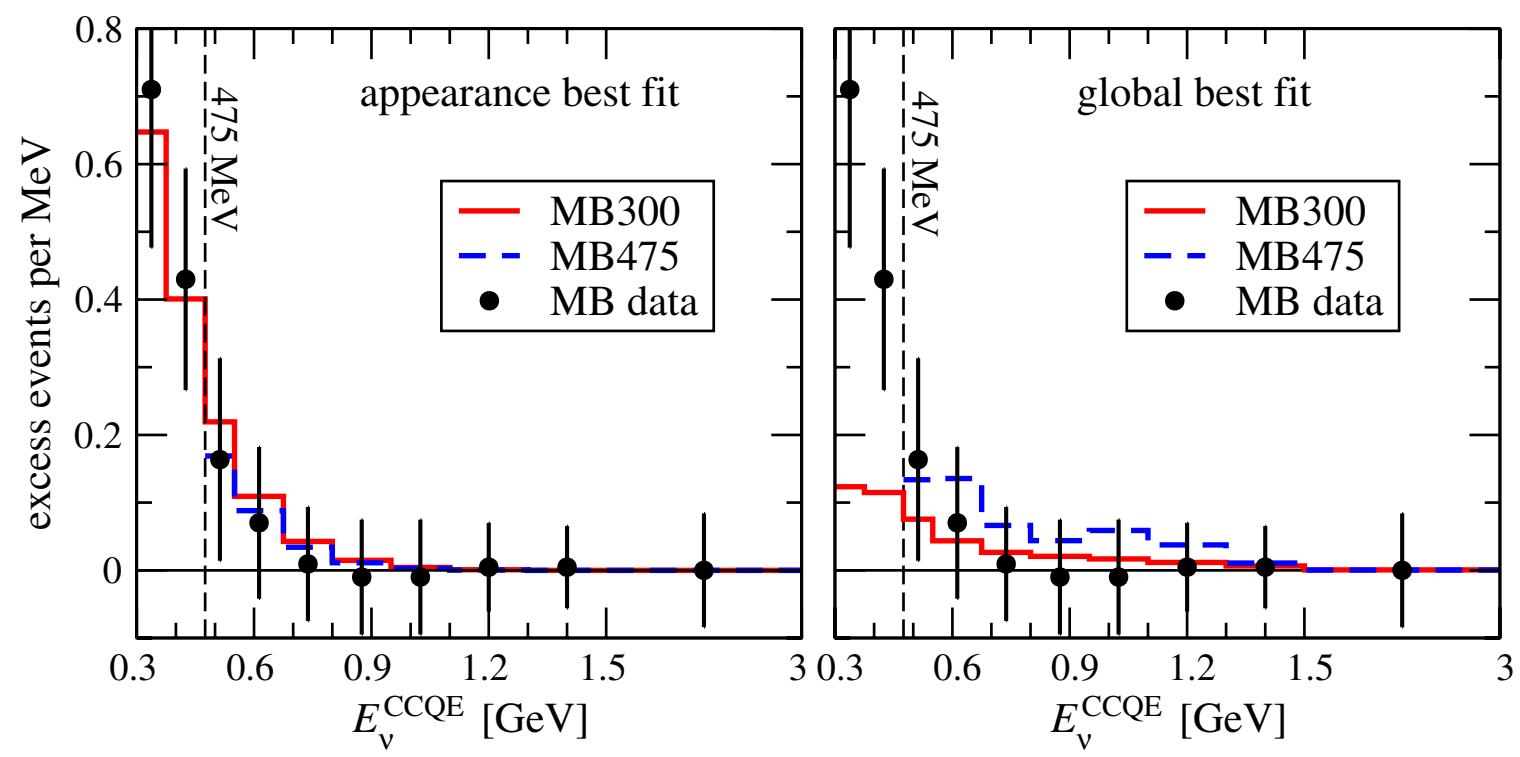

FIG. 3 (color online). MB spectral data in bins of reconstructed CCQE neutrino energy. The histograms show the prediction at the best fit points in $(3+2)$ mass schemes for SBL appearance data LSND, KARMEN, NOMAD, MB (left), and for the global data (right). For the solid histograms the full MB energy range has been used in the fit (MB300), whereas for the dashed histogram the two lowest energy data points have been omitted (MB475). The corresponding parameter values are given in Table I. 
TABLE I. Parameter values, $\chi^{2}$, and gof of the best fit points for SBL appearance data from LSND, KARMEN, NOMAD, MB (upper part), and global data (lower part) in $(3+2)$ schemes. Mass-squared differences are given in $\mathrm{eV}^{2}$. Results are shown without (MB475), and including (MB300) the low energy data from MB.

\begin{tabular}{|c|c|c|c|c|c|c|c|c|c|}
\hline Data set & \multicolumn{2}{|c|}{$\left|U_{e 4} U_{\mu 4}\right|$} & $\Delta m_{41}^{2}$ & \multicolumn{2}{|c|}{$\left|U_{e 5} U_{\mu 5}\right|$} & $\Delta m_{51}^{2}$ & $\delta$ & $\chi_{\min }^{2} /$ dof & gof \\
\hline Appearance (MB475) & \multicolumn{2}{|c|}{0.044} & 0.66 & \multicolumn{2}{|c|}{0.022} & 1.44 & $1.12 \pi$ & $16.9 /(29-5)$ & $85 \%$ \\
\hline \multirow[t]{2}{*}{ Appearance (MB300) } & \multicolumn{2}{|c|}{0.31} & 0.66 & \multicolumn{2}{|c|}{0.27} & 0.76 & $1.01 \pi$ & $18.5 /(31-5)$ & $85 \%$ \\
\hline & $\left|U_{e 4}\right|$ & $\left|U_{\mu 4}\right|$ & & $\left|U_{e 5}\right|$ & $\left|U_{\mu 5}\right|$ & & & & \\
\hline Global data (MB475) & 0.11 & 0.16 & 0.89 & 0.12 & 0.12 & 6.49 & $1.64 \pi$ & $94.5 /(107-7)$ & $63 \%$ \\
\hline Global data (MB300) & 0.12 & 0.18 & 0.87 & 0.11 & 0.089 & 1.91 & $1.44 \pi$ & $104.4 /(109-7)$ & $41 \%$ \\
\hline
\end{tabular}

and therefore, the total number of independent parameters is $\mathbf{5}$ if only appearance experiments are considered.

Nontrivial values of the complex phase $\delta$ lead to $C P$ violation, and hence in $(3+2)$ schemes much more flexibility is available to accommodate the results of LSND (antineutrinos) and MB (neutrinos). ${ }^{2}$ Indeed we find that MB is perfectly compatible with LSND in the $(3+2)$ framework. In Fig. 3 (left) we show the prediction for $\mathrm{MB}$ at the best fit points in the combined MB, LSND, KARMEN, NOMAD analysis. Clearly MB data can be fitted very well by simultaneously explaining the LSND evidence; we have checked that the prediction for the LSND oscillation probability is within the $1 \sigma$ range of the observed value. In this case also the low energy MB data can be explained, and therefore, in contrast to $(3+1)$ schemes, $(3+2)$ oscillations offer an appealing possibility to account for this excess. In the following we will present results from both MB data sets, MB475 as well as MB300. Note that for MB475 the number of data points used in our analysis is given in Eq. (2), whereas for the case of MB300 two more data points should be added to $N_{\mathrm{APP}}$ and $N_{\text {tot }}$. The parameter values and the $\chi^{2}$ minima at the best fit points are given in Table I. In both cases, MB475 and MB300, a gof of $85 \%$ is obtained, showing that MB is in very good agreement with global SBL appearance data including LSND.

In Fig. 4 (bottom) the $\chi^{2}$ is shown as a function of the $C P$ phase $\delta$. The data prefer values in the range $\pi<\delta<$ $2 \pi$ in order to reconcile LSND and MB. However, as visible in the figure no pronounced minimum appears and a rather broad range of $\delta$ values leads to a good fit, including also values rather close to the $C P$ conserving value $\delta=\pi$. For MB300 the best fit even occurs at $\delta=$ $1.01 \pi$; compare Table I. In Appendix A we give an explanation of how LSND and MB can be reconciled with values of $\delta$ so close to $\pi$. As discussed there, this mechanism is based on a delicate interplay of the three terms in the probability of Eq. (5), and a "tiny amount of $C P$

\footnotetext{
${ }^{2}$ The possibility to use $C P$ violation to reconcile LSND with a possible null result of $\mathrm{MB}$ neutrino data was pointed out in Ref. [31] in the framework of neutrino decay, and later in Ref. [50] in relation with $(3+2)$ oscillations.
}

violation" suffice to make LSND and MB compatible. However, this mechanism requires rather large values of the appearance amplitudes $\left|U_{e 4} U_{\mu 4}\right|$ and $\left|U_{e 5} U_{\mu 5}\right|$, which are not compatible with disappearance data, and hence solutions with $\delta$ close to $\pi$ are not possible in the global fit (see upper panel of Fig. 4 and the discussion in Sec. III B).

The allowed range in the plane of the two mass-squared differences is shown in Fig. 5. Again we observe that the solution is not particularly fine-tuned and a rather wide 90\% C.L. region appears. The fit using MB300 is somewhat more constrained by the requirement to fit the low energy excess in MB. The fact that for MB300 the best fit

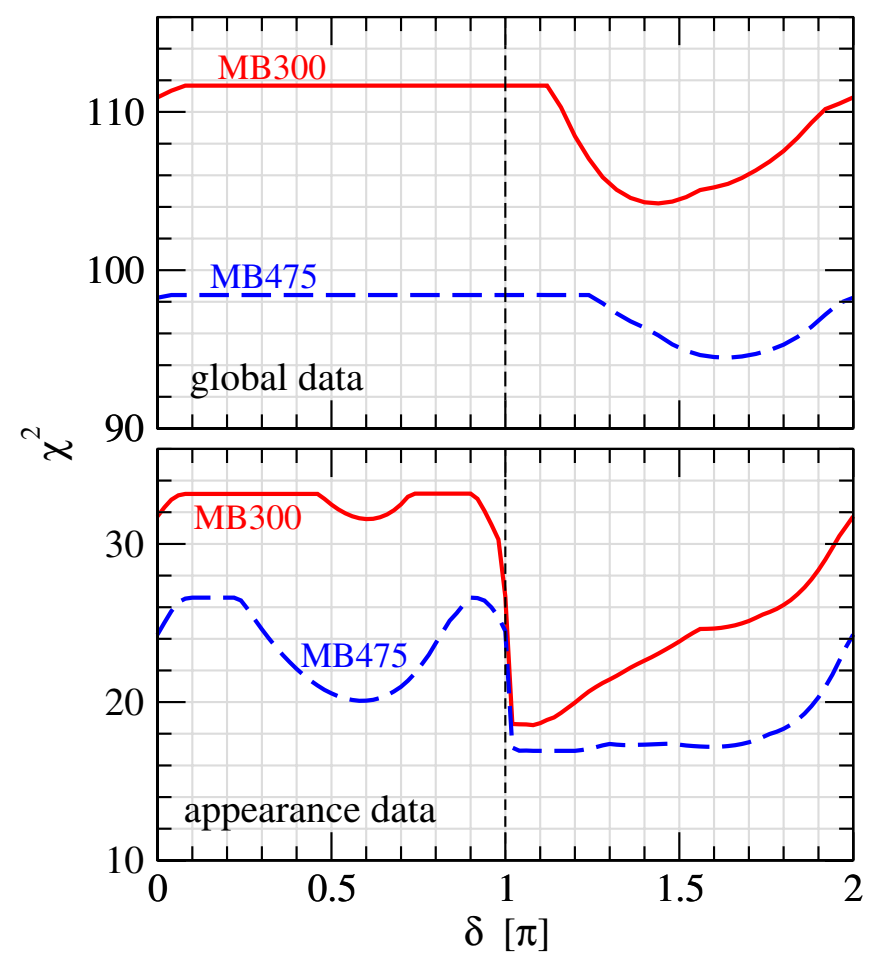

FIG. 4 (color online). The $\chi^{2}$ in $(3+2)$ schemes as a function of the $C P$ phase $\delta$ defined in Eq. (6) for appearance data from LSND, KARMEN, NOMAD, and MB (bottom), and for global data (top). Results are shown without (MB475), and including (MB300) the low energy data from MB. All other parameters have been minimized, respecting the constraint $\Delta m_{51}^{2} \geq \Delta m_{41}^{2}$. 

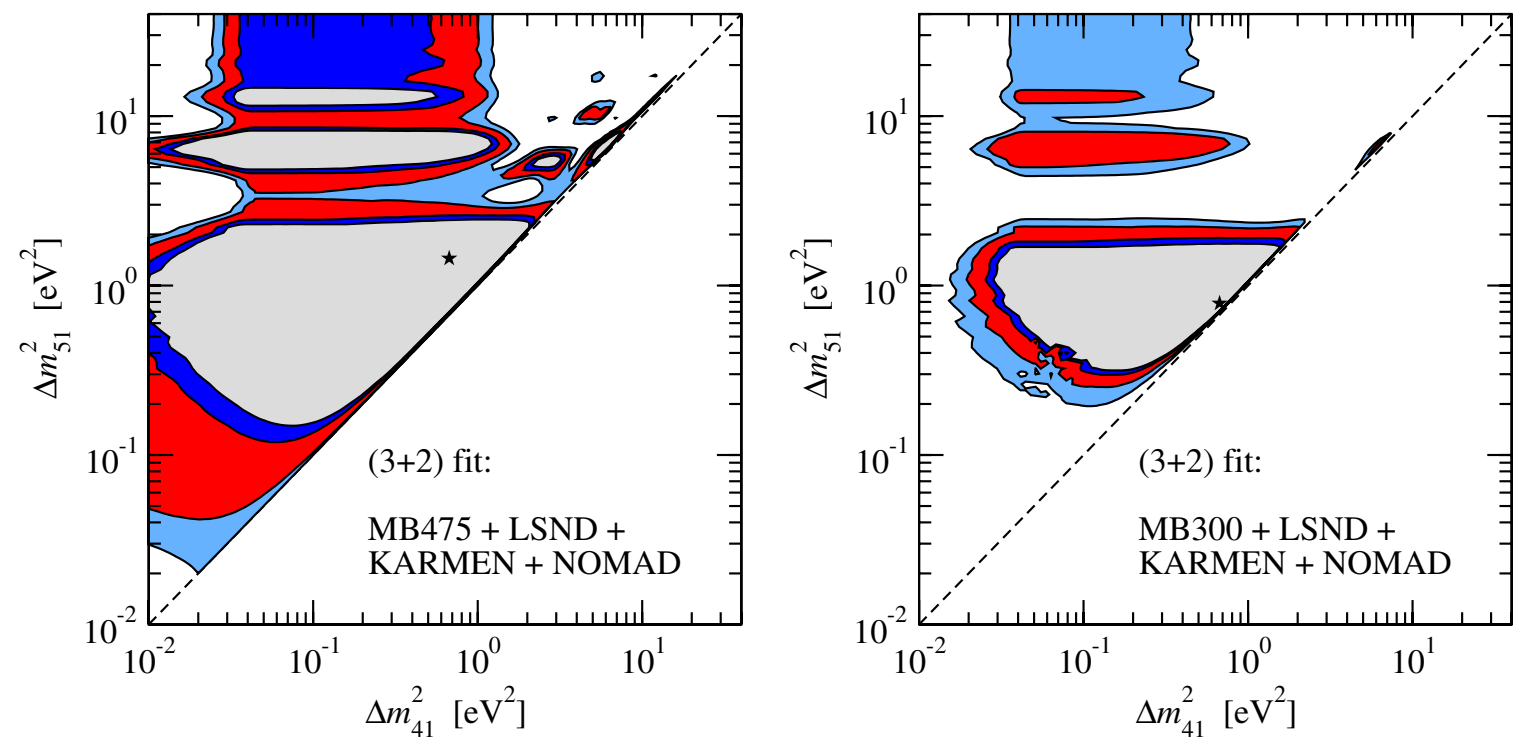

FIG. 5 (color online). Allowed regions for SBL appearance data in $(3+2)$ schemes at 90\%, 95\%, 99\%, 99.73\% C.L. (2 dof) in the plane of $\Delta m_{41}^{2}$ and $\Delta m_{51}^{2}$. All other parameters have been minimized. We use data from LSND, KARMEN, NOMAD, and MB475 (left) or MB300 (right).

occurs for $\Delta m_{41}^{2} \approx \Delta m_{51}^{2}$ is not statistically significant; the allowed region extends far into the hierarchical regime.

\section{B. Global SBL data in $(3+2)$ schemes}

Now we proceed to the global analysis in $(3+2)$ schemes, to see whether the successful description of all appearance data found in the previous subsection can be reconciled also with the bounds from disappearance experiments. The $(3+2)$ survival probability in the SBL approximation is given by

$$
\begin{aligned}
P_{\nu_{\alpha} \rightarrow \nu_{\alpha}}= & 1-4\left(1-\sum_{i=4,5}\left|U_{\alpha i}\right|^{2}\right) \sum_{i=4,5}\left|U_{\alpha i}\right|^{2} \sin ^{2} \phi_{i 1} \\
& -4\left|U_{\alpha 4}\right|^{2}\left|U_{\alpha 5}\right|^{2} \sin ^{2} \phi_{54},
\end{aligned}
$$

where $\phi_{i j}$ is given in Eq. (6). Similar to the $(3+1)$ case, also for $(3+2)$ schemes atmospheric neutrino data provide an important constraint on $\nu_{\mu}$ oscillations with sterile neutrinos. The five-neutrino atmospheric neutrino analysis is discussed in detail in Appendices B and C. It turns out that the same constraint $\chi_{\mathrm{ATM}}^{2}\left(d_{\mu}\right)$ as in the four-neutrino case applies, where now the definition $d_{\mu}=\left|U_{\mu 4}\right|^{2}+$ $\left|U_{\mu 5}\right|^{2}$ has to be used (see Appendix B).

The results of our global $(3+2)$ fit are summarized in the lower part of Table I, where the parameter values, the $\chi^{2}$, and the gof of the best fit points are given, again for both MB options, MB475 and MB300. The allowed regions for the global fit in the plane of the mass-squared differences are shown in Figs. 6 and 7.

In the right panel of Fig. 6 we show the $\chi^{2}$ projections for $(3+1)$ and $(3+2)$ schemes. Comparing the two best fit points provides a method to assess the relative quality of the fit in the two models (likelihood ratio test). We find that introducing the second sterile neutrino leads to the relative improvement of the fit of

$$
\chi_{\text {min,global (3+1) }}^{2}-\chi_{\text {min, global (3+2) }}^{2}=6.1 \quad(4 \mathrm{dof}),
$$

where the number of dof corresponds to the additional 4 parameters introduced by moving from $(3+1)$ to $(3+2)$. Hence, $(3+1)$ can be rejected only at the $81 \%$ C.L. with respect to the $(3+2)$ model. This explains also the "stripes" at $\Delta m_{41}^{2}$ and $\Delta m_{51}^{2}$ around $1 \mathrm{eV}^{2}$, which appear at $99 \%$ C.L. in Fig. 6. They correspond to the $(3+1)$ solution, which is always present as limiting case in $(3+$ 2). Also note, that in the case of appearance data alone we find

$$
\chi_{\min , \mathrm{APP}(3+1)}^{2}-\chi_{\min , \mathrm{APP}(3+2)}^{2}=9.7 \quad(3 \mathrm{dof}) .
$$

Comparing this number with Eq. (8) shows that the main improvement in $(3+2)$ schemes is to reconcile LSND and $\mathrm{MB}$, whereas it is not possible to evade efficiently the constraints from disappearance data. This result is somewhat in disagreement with the conclusion of Ref. [29]. A possible source of this different result might be the inclusion of atmospheric neutrino data in the fit, which is quite important to constrain sterile oscillations in the $\nu_{\mu}$ sector. Our results are in accordance with the arguments presented in the Appendix of Ref. [26].

In the upper panel of Fig. 4 the $\chi^{2}$ of the global fit is shown as a function of the complex phase $\delta$. One can see from that figure that the global data prefer values of $\delta$ close 

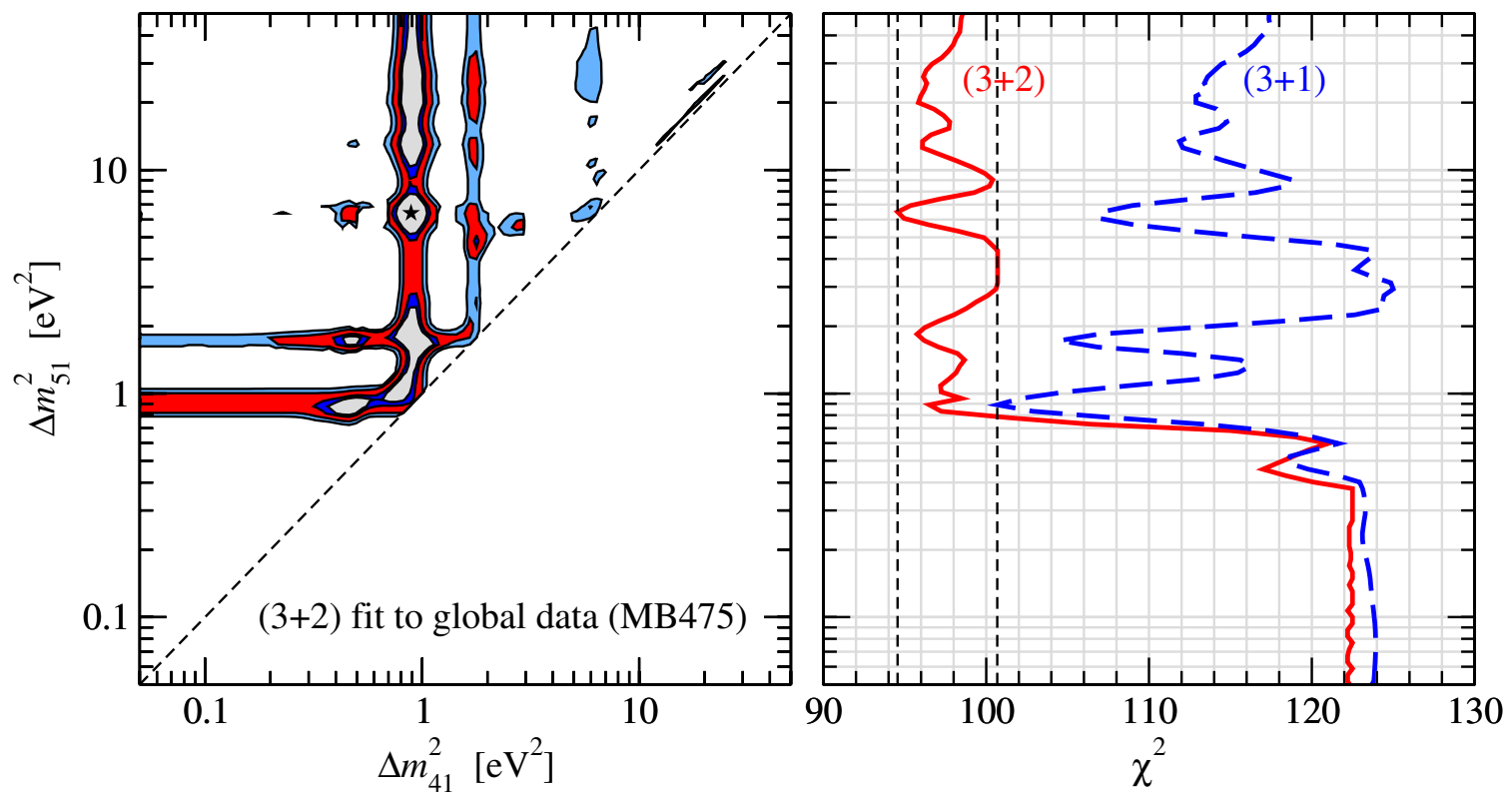

FIG. 6 (color online). Left: Allowed regions for global data in $(3+2)$ schemes at 90\%, 95\%, 99\%, 99.73\% C.L. (2 dof) in the plane of $\Delta m_{41}^{2}$ and $\Delta m_{51}^{2}$, with all other parameters minimized. Right: $\chi^{2}$ projected onto the $\Delta m_{51}^{2}$ axis, using $\Delta m_{41}^{2} \leq \Delta m_{51}^{2}$. For comparison we show also the $\chi^{2}$ projection in $(3+1)$ schemes. The vertical dashed lines indicate the $\chi^{2}$ of the $(3+2)$ and $(3+1)$ best fit points. For MB the two lowest energy data points have been omitted (MB475).

to "maximal" $C P$ violation at $\delta=3 \pi / 2$. The best fit values are $1.64 \pi$ and $1.44 \pi$ for MB475 and MB300, respectively. As discussed in Appendix A, reconciling MB and LSND with values of $\delta$ very close $\pi$ (as found for appearance data only) requires rather large values of the appearance amplitudes $\left|U_{e 4} U_{\mu 4}\right|$ and $\left|U_{e 5} U_{\mu 5}\right|$, close to the upper bound from unitarity. Such large values are not compatible with disappearance data, and hence solutions with $\delta$ close to $\pi$ are not possible in the global fit.

From the $\chi^{2}$ values given in Table I it appears that the model provides a very good fit to the data. However, as in the $(3+1)$ case the problem appears when the compatibility of different data sets is considered. Let us divide the global data into appearance and disappearance experi-
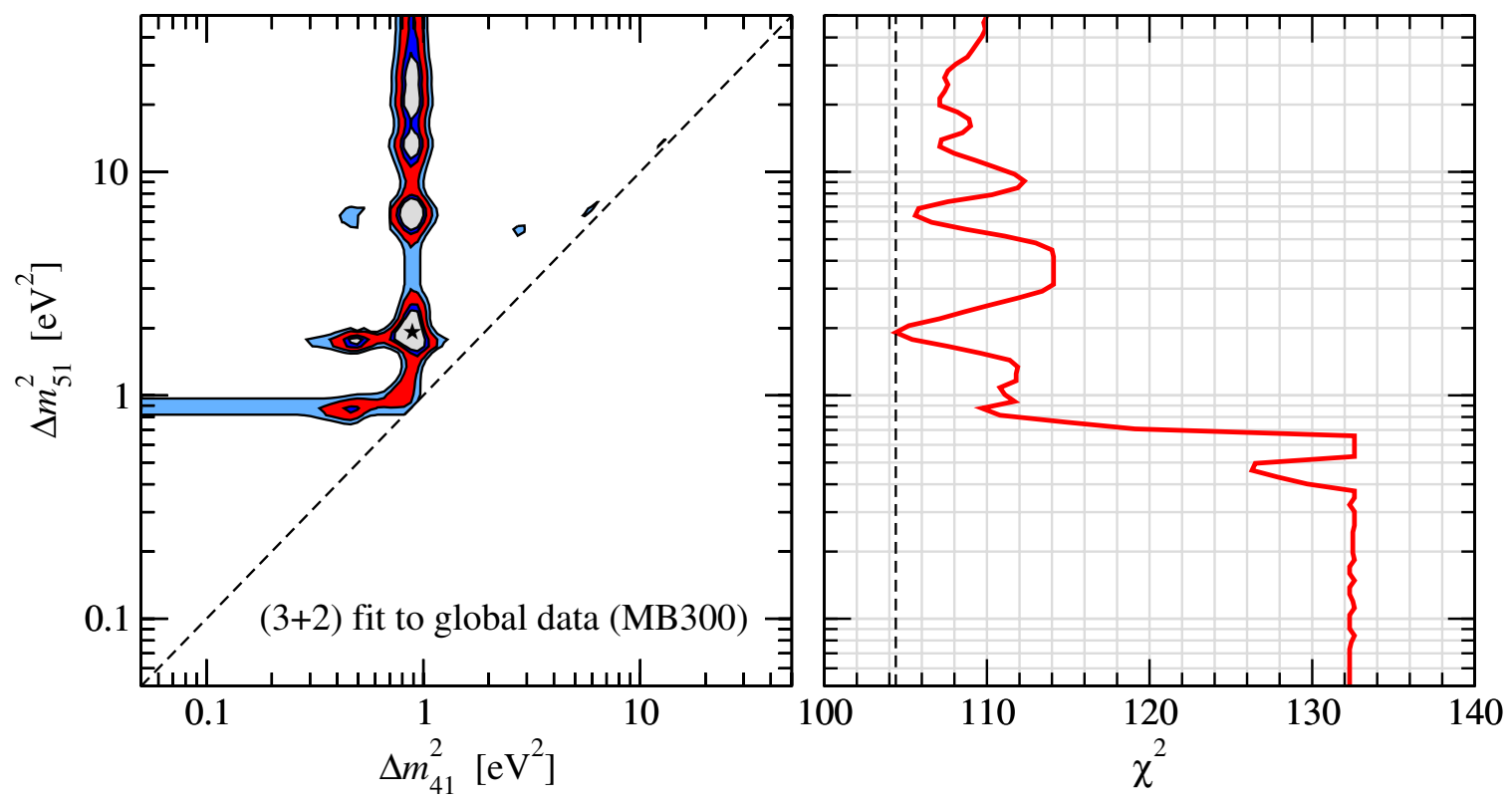

FIG. 7 (color online). Left: Allowed regions for global data in $(3+2)$ schemes at 90\%, 95\%, 99\%, 99.73\% C.L. (2 dof) in the plane of $\Delta m_{41}^{2}$ and $\Delta m_{51}^{2}$, with all other parameters minimized. Right: $\chi^{2}$ projected onto the $\Delta m_{51}^{2}$ axis, using $\Delta m_{41}^{2} \leq \Delta m_{51}^{2}$. The vertical dashed line indicates the $\chi^{2}$ of the best fit point. For MB the two lowest energy data points have been included (MB300). 

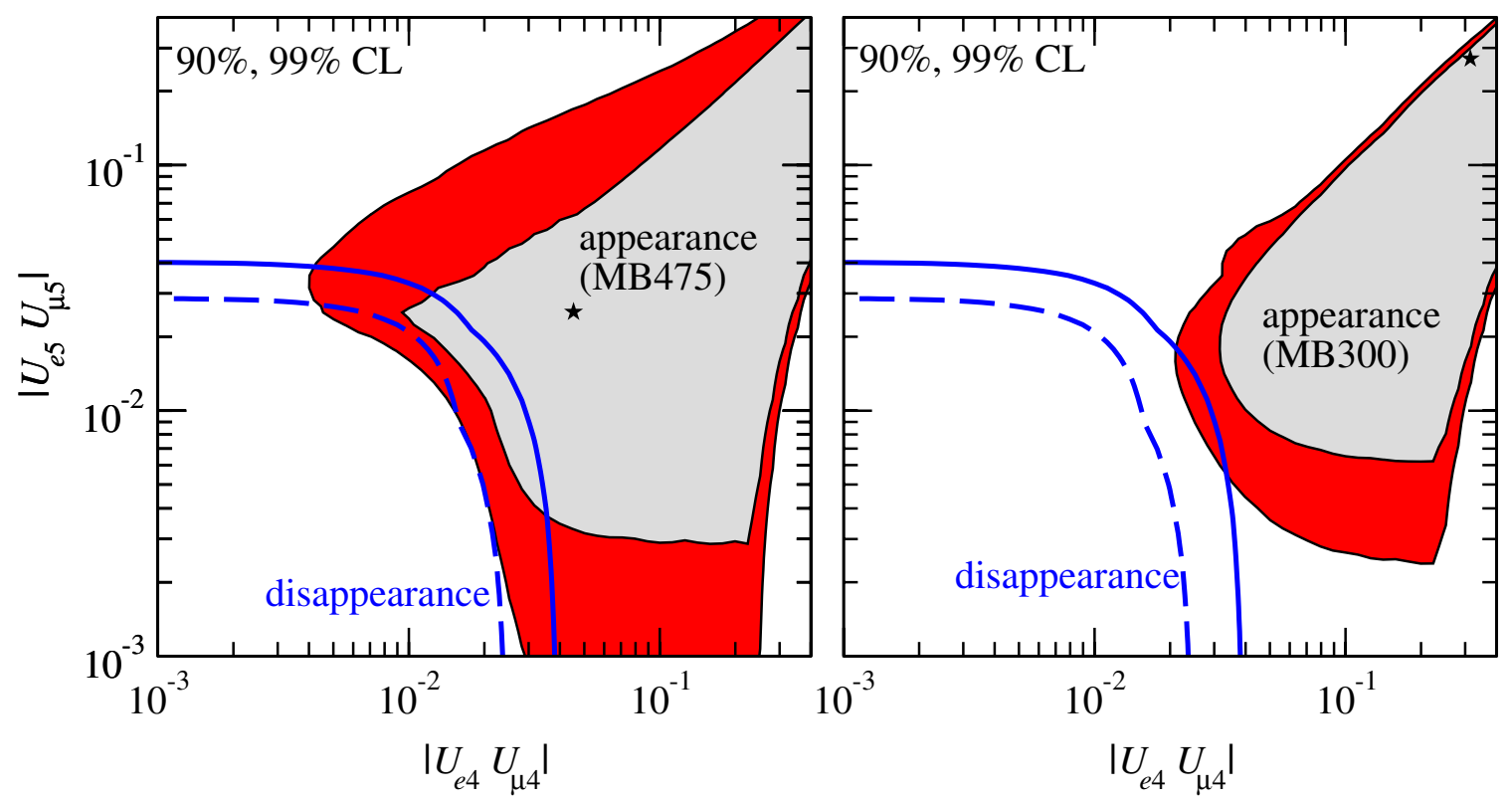

FIG. 8 (color online). Allowed regions at 90\% and 99\% C.L. in $(3+2)$ schemes for appearance data (shaded regions) and disappearance data (dashed curves and solid curves) projected onto the plane of $\left|U_{e 4} U_{\mu 4}\right|$ and $\left|U_{e 5} U_{\mu 5}\right|$. In the left panel the two lowest energy data points in MB have been omitted (MB475), whereas in the right panel the full MB energy range has been used in the fit (MB300).

ments and check their compatibility with the PG test [46] according to Eq. (1). We find the following $\chi_{\mathrm{PG}}^{2}$ values, for global data without MB, with MB475, and with MB300²:

$$
\begin{aligned}
& \chi_{\mathrm{PG}}^{2}=17.5, \quad \mathrm{PG}=1.5 \times 10^{-3} \quad(\text { no } \mathrm{MB}) \\
& \text { APP vs DIS: } \chi_{\mathrm{PG}}^{2}=17.2, \quad \mathrm{PG}=1.8 \times 10^{-3} \quad(\mathrm{MB} 475) \\
& \chi_{\mathrm{PG}}^{2}=25.1, \quad \mathrm{PG}=4.8 \times 10^{-5} \quad(\mathrm{MB} 300) \text {. }
\end{aligned}
$$

The PG values have been calculated for 4 dof [46]. This number corresponds to the four independent parameter (combinations) $\left|U_{e 4} U_{\mu 4}\right|,\left|U_{e 5} U_{\mu 5}\right|, \Delta m_{41}^{2}, \Delta m_{51}^{2}$, representing the minimal number of parameter (combinations) in common to the two data sets. From Eq. (10) we conclude that also in $(3+2)$ schemes a severe tension exists between appearance and disappearance experiments. If MB475 is used the result is very similar to the situation without MB data implying inconsistency at about $3.1 \sigma$, whereas in the case of the full MB data the tension becomes significantly worse (about $4 \sigma$ ), since appearance data are more constraining because of the need to accommodate LSND as well as the MB excess at low energies.

The tension between appearance and disappearance data is illustrated in Fig. 8, where we show the projections of the allowed regions in the plane of the appearance amplitudes $\left|U_{e 4} U_{\mu 4}\right|$ and $\left|U_{e 5} U_{\mu 5}\right|$. The opposite trend of the two data

\footnotetext{
${ }^{3}$ We have tested this result explicitly with the "official" MB analysis available at Ref. [45]. Using the MB $\chi^{2}$ from that source the PG test for appearance and disappearance data gives $\chi_{\mathrm{PG}}^{2}=$ 17.9 (MB475) and 24.6 (MB300), in good agreement with our results displayed in Eq. (10).
}

sets is clearly visible, especially when the low energy excess in MB is included (right panel). Note that an overlap of the regions visible in that figure does not prove that there is indeed an overlap of the allowed regions in the full parameters space since only a projection is shown. The "common" values in the plane shown in the plot might correspond actually to different locations in the space of $\Delta m_{41}^{2}$ and $\Delta m_{51}^{2}$. However, if no overlap is visible in that projection at a certain C.L. there is also no overlap at that C.L. in the full parameter space.

Comparing the numbers for MB475 and MB300 given in Eq. (10) it becomes obvious that the MB low energy excess is a severe problem in the global $(3+2)$ fit, although a very good fit can be obtained for appearance data only. This is also apparent from the $\chi_{\min }^{2}$ values given in Table I: Adding the two additional MB data points at low energy leads to an increase of the best fit $\chi^{2}$ of about 10 units from 94.5 to 104.4. Indeed, using the global data the MB excess cannot be fitted, as visible in the right panel of Fig. 3, where we show the prediction for the MB spectrum at the global best fit point. The reason is that to explain the excess relatively large values of $\left|U_{e 4} U_{\mu 4}\right|$ and $\left|U_{e 5} U_{\mu 5}\right|$ are required (see Fig. 8, right), which are inconsistent with disappearance data. 
Before closing this section we give the results of an alternative consistency test for the $(3+2)$ model. Instead of dividing the global data into appearance and disappearance experiments, we now consider the two data sets LSND and all the remaining NEV data, similar to what was done in Eq. (3) and Fig. 2 for the $(3+1)$ schemes. In the case of $(3+2)$ this analysis gives

$$
\chi_{\mathrm{PG}}^{2}=21.2(5 \mathrm{dof}), \quad \mathrm{PG}=7.8 \times 10^{-4}(3.4 \sigma)
$$

LSND vs NEV(incl. MB475).

Here 5 dof have been used, corresponding to the 5 parameters in common. Without $\mathrm{MB}$ we find in this case $\chi_{\mathrm{PG}}^{2}=$ $19.6, \mathrm{PG}=1.5 \times 10^{-3}(3.2 \sigma)$. Hence, the PG test gives a disagreement between LSND and the remaining SBL data similar to the disagreement between appearance and disappearance data found in Eq. (10).

\section{IV. $(3+3)$ SIX-NEUTRINO MASS SCHEMES}

Since there are three active neutrinos it seems natural to consider also the case of three sterile neutrinos. If all three additional neutrino states have masses in the $\mathrm{eV}$ range and mixings as relevant for the SBL experiments under consideration, such a model will certainly have severe difficulties to accommodate standard cosmology [40], and one has to refer to some nonstandard cosmological scenario [41-43]. Here we leave this problem aside and focus on neutrino oscillation data, investigating how much the fit improves with respect to the five-neutrino case.
The relevant oscillation probabilities are easily generalized to the $(3+3)$ scheme:

$$
\begin{aligned}
P_{\nu_{\mu} \rightarrow \nu_{e}}= & 4 \sum_{i}\left|U_{e i}\right|^{2}\left|U_{\mu i}\right|^{2} \sin ^{2} \phi_{i 1}+8 \sum_{i, j<i}\left|U_{e i} U_{\mu i} U_{e j} U_{\mu j}\right| \\
& \times \sin \phi_{i 1} \sin \phi_{j 1} \cos \left(\phi_{i j}-\delta_{i j}\right) \\
& i, j=4,5,6
\end{aligned}
$$

with the definitions

$$
\phi_{i j} \equiv \frac{\Delta m_{i j}^{2} L}{4 E}, \quad \delta_{i j} \equiv \arg \left(U_{e j}^{*} U_{\mu j} U_{e i} U_{\mu i}^{*}\right) .
$$

Equation (12) holds for neutrinos, for antineutrinos one has to replace $\delta_{i j} \rightarrow-\delta_{i j}$. Note that only two phases $\delta_{i j}$ are independent. The survival probability is given by

$$
\begin{aligned}
P_{\nu_{\alpha} \rightarrow \nu_{\alpha}}= & 1-4\left(1-\sum_{i}\left|U_{\alpha i}\right|^{2}\right) \sum_{i}\left|U_{\alpha i}\right|^{2} \sin ^{2} \phi_{i 1} \\
& -4 \sum_{i, j<i}\left|U_{\alpha i}\right|^{2}\left|U_{\alpha j}\right|^{2} \sin ^{2} \phi_{i j}, \quad i, j=4,5,6 .
\end{aligned}
$$

Atmospheric data is included in a similar way as in the previous cases, by using $\chi^{2}\left(d_{\mu}\right)$, where now we define $d_{\mu}=\sum_{i=4,5,6}\left|U_{\mu i}\right|^{2}$; see Appendix B. In general, one of the new mass splittings could also fall into the atmospheric range of few $\times 10^{-3} \mathrm{eV}^{2}$. We have not considered such degenerate situations, and we always assume that all $\Delta m_{i 1}^{2}$,
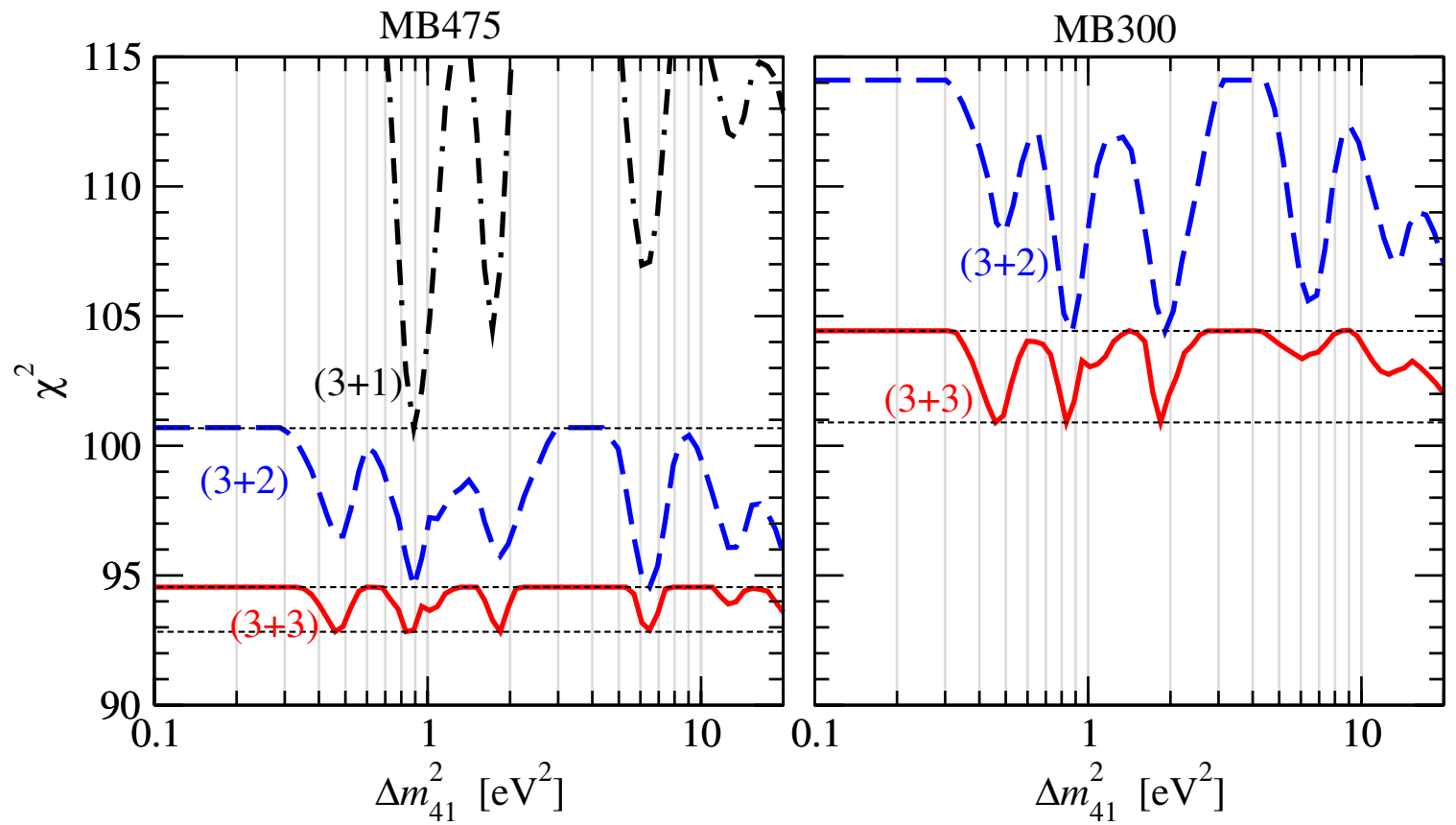

FIG. 9 (color online). $\quad \chi^{2}$ of global data in $(3+3)$ six-neutrino mass schemes projected onto the $\Delta m_{41}^{2}$ axis, using MB475 (left) or MB300 (right). For comparison also the $\chi^{2}$ is shown for the $(3+2)$ and $(3+1)$ schemes. The horizontal dashed lines indicate the corresponding best fit $\chi^{2}$ values. 
TABLE II. Best fit points for global data in the $(3+3)$ scheme. Mass-squared differences are given in $\mathrm{eV}^{2}$. We give also the $\chi^{2}$ difference between the $(3+2)$ and $(3+3)$ best fits. The last column shows the C.L. at which $(3+2)$ is accepted with respect to $(3+3)$, as derived from the $\chi^{2}$ difference evaluated for 4 dof, corresponding to four additional parameters in the $(3+3)$ model.

\begin{tabular}{cccccccc}
\hline \hline & $\Delta m_{41}^{2}$ & $\Delta m_{51}^{2}$ & $\Delta m_{61}^{2}$ & $\chi_{\min }^{2} /$ dof & gof & $\chi_{(3+2)}^{2}-\chi_{(3+3)}^{2}$ & C.L. \\
\hline MB475 & 0.46 & 0.83 & 1.84 & $92.8 /(107-11)$ & $57 \%$ & $1.7 / 4$ & $20 \%$ \\
MB300 & 0.46 & 0.83 & 1.84 & $100.9 /(109-11)$ & $40 \%$ & $3.5 / 4$ & $52 \%$ \\
\hline \hline
\end{tabular}

$\Delta m_{i j}^{2}$, with $i, j=4,5,6$ are infinite for the atmospheric neutrino analysis.

We have scanned the $\chi^{2}$ of global data in the 11 dimensional parameter space, where a grid of $81 \times 81 \times 81$ values for the mass-squared differences has been used, spaced logarithmically from 0.1 to $20 \mathrm{eV}^{2}$. In each point of this grid the remaining 8 parameters have been minimized by a standard optimization routine.

The results of our search are shown in Fig. 9, where we display the global $\chi^{2}$ as a function of $\Delta m_{41}^{2}$. For comparison also the $\chi^{2}$ curves in the case of $(3+2)$ and for MB475 also for $(3+1)$ are shown. Note that different from Sec. III, here we do not impose any constraint on the ordering of the mass-squared differences, and the full $s$-dimensional space is scanned for the $(3+s)$ scheme. This implies that the result is symmetric with respect to the $s$ mass-squared differences, and the $\chi^{2}$ functions projected onto any of the $\left(\Delta m_{i 1}^{2}\right)$ axes $(i \geq 4)$ are identical. (Just for convenience we label the horizontal axis in Fig. 9 as $\Delta m_{41}^{2}$, since it is available in all schemes.) Furthermore, as a consequence of this symmetry there are 2 (3) degenerate minima in the five (six) neutrino case, which corresponds to a relabelling of the mass states. Note also, as it must be, the $\chi_{\min }^{2}$ of the $(3+(s-1))$ model is the maximal $\chi^{2}$ value in $(3+s)$, since the $(3+(s-1))$ fit is always available as limiting case.

The global $(3+3)$ best fit points are summarized in Table II. From the table and Fig. 9 one can see that there is only a marginal improvement of the fit by 1.7 units in $\chi^{2}$ for MB475 (3.5 for MB300) with respect to $(3+2)$, to be compared with 4 additional parameters in the model. Hence, we conclude that there are no qualitatively new effects in the $(3+3)$ scheme. The conflict between appearance and disappearance data remains a problem, and the additional freedom introduced by four new parameters does not relax significantly this tension.

\section{SUMMARY}

We have considered the global fit to SBL neutrino oscillation data including the recent data from the MiniBooNE (MB) experiment [1] in the framework of four-, five-, and six-neutrino oscillations. We have divided the global data into various subsets and tested their consistency within the sterile-neutrino oscillation framework.
These results are summarized in Table III for the $(3+1)$ and $(3+2)$ schemes. Clearly, in all cases we find severe tension between different subsamples of the data, with the only exception when LSND and the low-energy excess in $\mathrm{MB}$ are left out, and in this case indeed no sterile neutrinos are needed and the standard three active neutrino scheme $(3+0)$ provides a perfect fit to the data.

Let us summarize our findings:

(1) $(3+1)$ four-neutrino schemes are strongly disfavored because

(a) recent MB data is incompatible with LSND at the $98 \%$ C.L. [1]

(b) the tension between LSND and NEV SBL data becomes more severe due to $\mathrm{MB}$, there is no overlap of the allowed regions for NEV and LSND at 99\% C.L., and the PG test implies inconsistency at the level of $4 \sigma$,

(c) it is not possible to account for the low energy event excess in MB.

(2) $(3+2)$ five-neutrino schemes

(a) do provide a good fit to LSND and the recent MB data,

(b) they can account for the low energy event excess in $\mathrm{MB}$, however

(c) there is significant tension between appearance and disappearance data (according to the PG test at the level of $3 \sigma$ for MB475 and $4 \sigma$ for MB300).

(3) $(3+3)$ six-neutrino schemes do not offer qualitatively new effects, the global $\chi^{2}$ improves only by about 1.7 (3.5) units for MB475 (MB300) with respect to $(3+2)$, and hence, the conflict between appearance and disappearance data remains.

The points $2 \mathrm{a}$ and $2 \mathrm{~b}$ might be considered as an interesting hint in favor of $(3+2)$ schemes. Since the combined fit of LSND and MB is based on a nontrivial complex phase which introduces a difference in neutrino and antineutrino oscillations, these results would represent the first indication of $C P$ violation in neutrino oscillations. This hypothesis could be tested by MB antineutrino data, which is currently being accumulated. However, point $2 \mathrm{c}$ is a challenge for the $(3+2)$ model. The conclusions of $2 \mathrm{c}$ and 3 strongly rely on the disappearance experiments Bugey and CDHS. A crucial check would be the confirmation of $\nu_{e}$ or 
TABLE III. Summary table for various consistency checks within the $(3+1)$ and $(3+2)$ schemes. The PG test [46] has been defined in Eq. (1). In the first column, where we give the data sets tested against each other, we use the following abbreviations: K $($ KARMEN), N (NOMAD), L (LSND), DIS corresponds to the disappearance experiments summarized in Eq. (2), and NEV = DIS $+K+N$. Results for the $(3+3)$ scheme are qualitatively similar to $(3+2)$.

\begin{tabular}{|c|c|c|c|c|c|c|}
\hline & $\chi_{\text {global }}^{2} /$ dof & $\begin{array}{c}(3+1) \\
\chi_{\mathrm{PG}}^{2} / \mathrm{dof}\end{array}$ & PG & $\chi_{\text {global }}^{2} /$ dof & $\begin{array}{c}(3+2) \\
\chi_{\mathrm{PG}}^{2} / \mathrm{dof}\end{array}$ & PG \\
\hline DIS vs $\mathrm{K}+\mathrm{N}+\mathrm{L}$ & $95.5 / 96$ & $14.8 / 2$ & $6.1 \times 10^{-4}$ & $92.1 / 92$ & $17.4 / 4$ & $1.5 \times 10^{-3}$ \\
\hline DIS vs $\mathrm{K}+\mathrm{N}+\mathrm{L}+\mathrm{MB} 475$ & & & & $94.5 / 100$ & $17.2 / 4$ & $1.8 \times 10^{-3}$ \\
\hline DIS vs $\mathrm{K}+\mathrm{N}+\mathrm{L}+\mathrm{MB} 300$ & & & & $104.4 / 102$ & $25.1 / 4$ & $4.8 \times 10^{-5}$ \\
\hline DIS vs $\mathrm{K}+\mathrm{N}+\mathrm{MB} 475$ & $70.5 / 93$ & $0.1 / 2$ & 0.95 & $68.9 / 89$ & $1.1 / 4$ & 0.89 \\
\hline DIS vs $\mathrm{K}+\mathrm{N}+\mathrm{MB} 300$ & & & & $79.1 / 91$ & $10.3 / 4$ & $3.6 \times 10^{-2}$ \\
\hline NEV vs L & $95.5 / 96$ & $20.9 / 2$ & $2.9 \times 10^{-5}$ & $92.1 / 92$ & $19.6 / 5$ & $1.5 \times 10^{-3}$ \\
\hline $\mathrm{NEV}+\mathrm{MB} 475$ vs $\mathrm{L}$ & $100.7 / 104$ & $24.7 / 2$ & $4.3 \times 10^{-6}$ & $94.5 / 100$ & $21.2 / 5$ & $7.8 \times 10^{-4}$ \\
\hline
\end{tabular}

$\nu_{\mu}$ disappearance at the $1 \mathrm{eV}^{2}$ scale. Hence, it might be worthwhile to investigate the possibility to obtain such information at future reactor experiments [51], from disappearance experiments based on low-energy neutrinos from radioactive sources [52], or at the near detector complex of upcoming long-baseline superbeam experiments [53]. A characteristic signal of sterile neutrino oscillations could be obtained at experiments exploring neutral-current detection [54].

\section{ACKNOWLEDGMENTS}

We thank Michel Sorel for communication on the MiniBooNE experiment and useful comments on our analysis. M. M. is supported by MCYT through the Ramón y Cajal program, by CiCYT through the project FPA2006-01105, and by the Comunidad Autónoma de Madrid through the project P-ESP-00346.

\section{APPENDIX A: RECONCILING LSND AND MB IN $(3+2)$ SCHEMES}

In this Appendix we discuss in some detail how LSND and $\mathrm{MB}$ are reconciled in $(3+2)$ schemes exploring $C P$ violation in the appearance probability. In particular, it is intriguing that a very good fit can be obtained with a complex phase $\delta$ very close to the $C P$ conserving value $\pi$; compare Fig. 4. To understand this effect we show in Fig. 10 a zoom into the region around $\delta=\pi$, and we display in addition to the $\chi^{2}$ also the values obtained for the oscillation parameters.

Let us consider the probability $P_{\nu_{\mu} \rightarrow \nu_{e}}$ given in Eq. (5). A nontrivial possibility to suppress this probability can be obtained by requiring $\cos \left(\phi_{54}-\delta\right)=-1$. Then one has

$$
P_{\nu_{\mu} \rightarrow \nu_{e}}=4\left(q_{4} \sin \phi_{41}-q_{5} \sin \phi_{51}\right)^{2},
$$

$\cos \left(\phi_{54}-\delta\right)=-1$,

with the abbreviation $q_{i} \equiv\left|U_{e i} U_{\mu i}\right|$. Hence, the probability is small for $q_{4} \approx q_{5}$ and $\phi_{54} \ll 1$. This is precisely the behavior shown in Fig. 10: when $\delta$ approaches $\pi$ from above, $\Delta m_{54}^{2}$ becomes small and the $q_{i}$ approach each other. Writing $\delta=\pi+\epsilon$ one has $\cos \left(\phi_{54}-\delta\right) \approx-1+$ $\mathcal{O}\left(\phi_{54}^{2}, \epsilon^{2}\right)$, Eq. (A1) is valid, and the oscillation probability is suppressed in MB.

Now the question arises whether large enough values for $P_{\bar{\nu}_{\mu} \rightarrow \bar{\nu}_{e}}$ can be achieved in order to explain LSND. The difference of antineutrino and neutrino probabilities is given by

$$
\begin{aligned}
P_{\bar{\nu}_{\mu} \rightarrow \bar{\nu}_{e}}-P_{\nu_{\mu} \rightarrow \nu_{e}} & =16 q_{4} q_{5} \sin \phi_{41} \sin \phi_{51} \sin \phi_{54} \sin \epsilon \\
& \approx 16 q_{4} q_{5} \sin ^{2}\left(\phi_{51}\right) \phi_{54} \epsilon,
\end{aligned}
$$

where in the last step $\phi_{54}, \epsilon \ll 1$ has been used. Since $\phi_{54}$ and $\epsilon$ are small, the other factors have to be as large as possible in order to get a sufficient probability for LSND. Indeed, for $\Delta m_{51}^{2} \approx 1 \mathrm{eV}^{2}$ one has $\sin ^{2} \phi_{51} \approx 1$, and also the $q_{i}$ grow for $\epsilon \rightarrow 0$ (see Fig. 10). Once the maximal values allowed by unitarity, $q_{4}=q_{5}=1 / 2$, are reached the LSND probability is given roughly by $P_{\bar{\nu}_{\mu} \rightarrow \bar{\nu}_{e}} \sim 4 \epsilon^{2}$, where we used $P_{\nu_{\mu} \rightarrow \nu_{e}} \approx 0$ (in order to explain MB) and $\phi_{54} \approx \epsilon$ [in order to have $\left.\cos \left(\phi_{54}-\delta\right) \approx-1\right]$. Using the experimental value $P_{\text {LSND }}=0.0026$ one finds that a fit should be possible for $\epsilon \gtrsim 0.025 \approx 0.008 \pi$, in agreement with our results.

The similar structure of the left and right panels of Fig. 10 suggests that this mechanism works equally well for MB475 and MB300, and fitting the low energy excess in $\mathrm{MB}$ does not affect these considerations. Obviously, this explanation is not valid for $\delta<\pi$, since the $C P$ asymmetry Eq. (A2) has the wrong sign to reconcile LSND and MB. As visible in Fig. 10, the fit jumps into a quite different solution, which anyway gives a poor $\chi^{2}$. Also, the local minimum around $\delta \sim \pi / 2$ visible in Fig. 4 for MB475 requires a different explanation in order to obtain the correct sign of the $C P$ asymmetry for these values of $\delta$. Let us also mention that quite large values of $q_{4}$ and $q_{5}$ close to the unitarity bound do appear in the fit for $\delta \gtrsim \pi$, since only appearance experiments are used. Such large values are not possible if disappearance experiments are included, which basically require that each of the $\left|U_{e i}\right|$, 


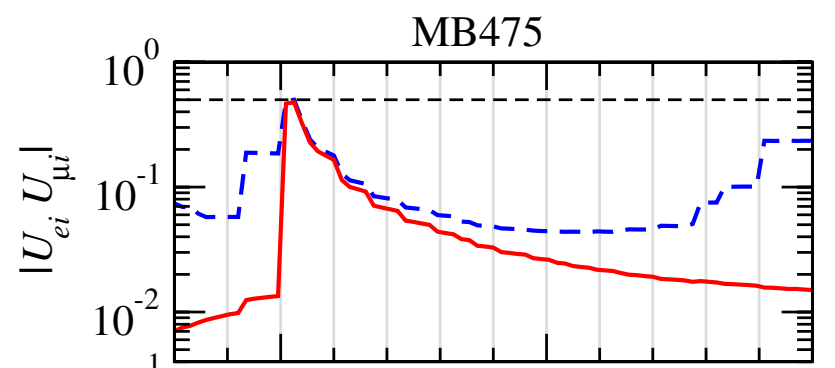

MB300
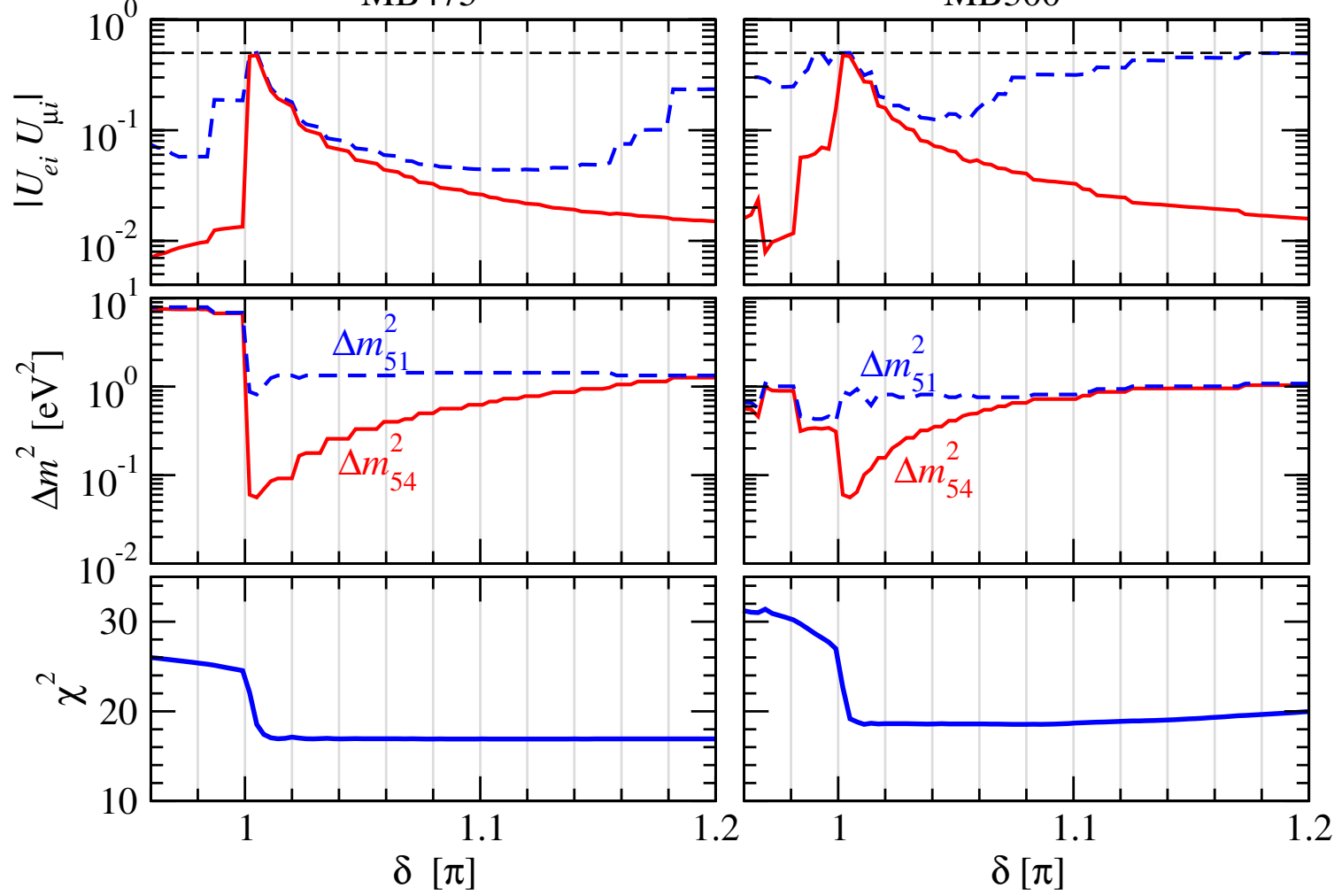

FIG. 10 (color online). Fit to appearance data LSND, KARMEN, NOMAD, and MB475 (left) or MB300 (right) in (3 + 2) schemes. We show the $\chi^{2}$ (bottom), the values of $\Delta m_{51}^{2}, \Delta m_{54}^{2}$ (middle), and the values of $q_{i} \equiv\left|U_{e i} U_{\mu i}\right|, i=4,5$ (top) as a function of the complex phase $\delta$ defined in Eq. (6). The horizontal dashed lines in the top panels correspond to the maximal value allowed by unitarity of the mixing matrix.

$\left|U_{\mu i}\right|, i=4,5$ has to be small. This is one reason for the difficulties in reconciling appearance and disappearance data, in close analogy to $(3+1)$.

\section{APPENDIX B: OSCILLATIONS WITH EXTRA STERILE STATES}

In this Appendix we discuss in some detail atmospheric and short-baseline neutrino oscillations involving extra sterile neutrino states. For definiteness, we will focus on $(3+3)$ schemes; expressions for $(3+2)$ and $(3+1)$ models can be easily obtained by dropping all terms containing a redundant " 6 " or " 5 " index. Let us order the flavor eigenstates as $\left(\nu_{e}, \nu_{\mu}, \nu_{\tau}, \nu_{s_{1}}, \nu_{s_{2}}, \nu_{s_{3}}\right)$ and introduce the following parametrization for the neutrino mixing:

$$
U=\tilde{R}_{36} \tilde{R}_{35} \tilde{R}_{34} R_{26} R_{25} R_{24} R_{23} \tilde{R}_{16} \tilde{R}_{15} R_{14} \tilde{R}_{13} \tilde{R}_{12}
$$

where $\tilde{R}_{i j}$ represents a complex rotation by an angle $\theta_{i j}$ and a phase $\varphi_{i j}$ in the $i j$ plane, while $R_{i j}$ is an ordinary rotation by an angle $\theta_{i j}$. Note that rotations involving only sterile states (i.e., $\tilde{R}_{\ell \ell^{\prime}}$ with both $\ell, \ell^{\prime} \geq 4$ ) are unphysical, and therefore we have omitted them from Eq. (B1). For the general case with $s$ sterile states, it is convenient to choose the $(s+2)$ rotations $R_{14}$ and $R_{2 j}$ as real, and the remaining ones as complex. The matrix $U$ then includes $3(s+1)$ angles and $(2 s+1)$ phases.

A number of simplifying assumptions can be made in the analysis of short-baseline as well as atmospheric and long-baseline neutrino experiments. For short-baseline experiments one can neglect the solar and atmospheric mass splittings, $\Delta m_{21}^{2}=0$ and $\Delta m_{31}^{2}=0$. In this approximation, the mixing angles $\tilde{\theta}_{12}, \tilde{\theta}_{13}$, and $\theta_{23}$ disappear from the relevant probabilities. Furthermore, matter effects can be neglected. Since we do not consider neutral current interactions in our analysis, the $\tau$ neutrino is essentially indistinguishable from the sterile states, as it participates neither in the production nor in the detection processes. Therefore, all the angles $\tilde{\theta}_{3 j}$ also disappear. So for $(3+3)$ models we are left with an effective mixing matrix

$$
U=R_{26} R_{25} R_{24} \tilde{R}_{16} \tilde{R}_{15} R_{14} \quad \text { for SBL, }
$$

which contains six angles and two $C P$ phases. In general, under our approximations SBL experiments depend on $2 s$ angles and $(s-1)$ phases. For example, in $(3+2)$ models we have four angles $\left(\theta_{14}, \theta_{15}, \theta_{24}, \theta_{25}\right)$ and one phase $\left(\varphi_{15}\right)$, and the matrix elements $\left|U_{e 4}\right|,\left|U_{e 5}\right|,\left|U_{\mu 4}\right|,\left|U_{\mu 5}\right|$, $\arg \left(U_{e 4}^{*} U_{\mu 4} U_{e 5} U_{\mu 5}^{*}\right)$ used in Sec. III are combinations of these five parameters. 
For atmospheric and long-baseline experiments (K2K and MINOS) we neglect the mixing of $\nu_{e}$ with other neutrino states at the LSND mass-squared splittings, justified by the constraint from Bugey. This corresponds to setting all the angles $\tilde{\theta}_{1 \ell}$ with $\ell \geq 4$ to zero. In this approximation, the complex phase $\varphi_{12}$ can be dropped. Therefore, in $(3+3)$ models we are left with an effective mixing matrix

$U=\tilde{R}_{36} \tilde{R}_{35} \tilde{R}_{34} R_{26} R_{25} R_{24} R_{23} \tilde{R}_{13} R_{12} \quad$ for ATM and LBL,

which contains nine angles and four $C P$ phases. As a general rule, in our approximation for ATM and LBL experiments the matrix $U$ contains $(2 s+3)$ angles and $(s+1)$ phases.

From Eqs. (B2) and (B3) it is straightforward to see that for any number of extra sterile states, $s$, atmospheric and short-baseline experiments are connected only through the $s$ angles $\theta_{2 \ell}$ with $\ell \geq 4$ (or, equivalently, through the parameters $\left|U_{\mu \ell}\right|^{2}$ with $\ell \geq 4$ ). Note that in our convention all the nonvanishing $C P$ phases are "private" to either short-baseline (e.g., $\varphi_{15}$ and $\varphi_{16}$ ) or atmospheric (e.g., $\varphi_{13}, \varphi_{34}, \varphi_{35}$, and $\left.\varphi_{36}\right)$ experiments.

Let us now focus on the probabilities relevant for the analysis of atmospheric and long-baseline experiments. The Hamiltonian in the flavor basis is

$$
H=U \Delta U^{\dagger}+V
$$

where $U$ is given in Eq. (B3), $\Delta=$ $\operatorname{diag}\left(0, \Delta m_{21}^{2}, \Delta m_{31}^{2}, \Delta m_{41}^{2}, \Delta m_{51}^{2}, \ldots\right) / 2 E, \quad$ and $\quad V=$ $\pm \sqrt{2} G_{F} \operatorname{diag}\left(2 N_{e}, 0,0, N_{n}, N_{n}, \ldots\right) / 2$. It is convenient to define $U_{\mathrm{SM}}=R_{23} \tilde{R}_{13} R_{12}$ and $\tilde{U}=U U_{\mathrm{SM}}^{\dagger}$. Then we can write:

$$
H=\tilde{U} \tilde{H} \tilde{U}^{\dagger} \quad \text { with } \quad \tilde{H}=U_{\mathrm{SM}} \Delta U_{\mathrm{SM}}^{\dagger}+\tilde{U}^{\dagger} V \tilde{U} .
$$

In order to further simplify the analysis, let us now assume that all the mass-squared differences involving the "heavy" states $\nu_{\ell}$ with $\ell \geq 4$ can be considered as infinite: $\Delta m_{\ell i}^{2}, \Delta m_{\ell \ell^{\prime}}^{2} \rightarrow \infty$ for any $i=1,2,3$ and $\ell, \ell^{\prime} \geq 4$. In leading order, the matrix $\tilde{H}$ takes the effective blockdiagonal form:

$$
\tilde{H} \approx\left(\begin{array}{cc}
H^{(3)} & \mathbf{0} \\
\mathbf{0} & \Delta^{(s)}
\end{array}\right)
$$

where $H^{(3)}$ is the $3 \times 3$ submatrix of $\tilde{H}$ corresponding to the first, second, and third neutrino states, and $\Delta^{(s)}=$ $\operatorname{diag}\left(\Delta m_{41}^{2}, \Delta m_{51}^{2}, \ldots\right) / 2 E$ is a diagonal $s \times s$ matrix (the matter terms in this block are negligible in the limit of very large $\Delta m_{\ell \ell^{\prime}}^{2}$. Consequently, the evolution matrix is

$$
\tilde{S} \approx\left(\begin{array}{cc}
e^{i H^{(3)} L} & \mathbf{0} \\
\mathbf{0} & e^{i \Delta^{(s)} L}
\end{array}\right) \quad \text { and } \quad S=\tilde{U} \tilde{S} \tilde{U}^{\dagger}
$$

We are interested only in the elements $S_{e e}, S_{e \mu}, S_{\mu e}$, and $S_{\mu \mu}$. Taking into account the block-diagonal form of $\tilde{S}$ and the relations $\tilde{U}_{e i}=\delta_{i 1}$ and $\tilde{U}_{\mu 1}=\tilde{U}_{\mu 3}=0$, we obtain:

$$
\begin{gathered}
S_{e e}=\tilde{S}_{11}, \quad S_{e \mu}=\tilde{U}_{\mu 2}^{\star} \tilde{S}_{12}, \quad S_{\mu e}=\tilde{U}_{\mu 2} \tilde{S}_{21}, \\
S_{\mu \mu}=\left|\tilde{U}_{\mu 2}\right|^{2} \tilde{S}_{22}+\sum_{\ell \geq 4}\left|\tilde{U}_{\mu \ell}\right|^{2} e^{i \Delta_{\ell \ell} L} .
\end{gathered}
$$

The expressions for the probabilities, $P_{\alpha \beta}=\left|S_{\alpha \beta}\right|^{2}$, are straightforward. Defining

$$
d_{\mu} \equiv \sum_{\ell \geq 4}\left|U_{\mu \ell}\right|^{2}
$$

we note that $\left|\tilde{U}_{\mu 2}\right|^{2}+\sum_{\ell \geq 4}\left|\tilde{U}_{\mu \ell}\right|^{2}=1$ and that $U_{\alpha \ell}=$ $\tilde{U}_{\alpha \ell}$ for $\ell \geq 4$, so that $\left|\tilde{U}_{\mu 2}\right|^{2}=1-d_{\mu}$. Therefore,

$$
\begin{gathered}
P_{e e}=P_{e e}^{(3)}, \quad P_{e \mu}=\left(1-d_{\mu}\right) P_{e \mu}^{(3)}, \\
P_{\mu e}=\left(1-d_{\mu}\right) P_{\mu e}^{(3)}, \\
P_{\mu \mu}=\left(1-d_{\mu}\right)^{2} P_{\mu \mu}^{(3)}+\sum_{\ell \geq 4}\left|U_{\mu \ell}\right|^{4},
\end{gathered}
$$

where we have used the fact that the terms containing a factor $e^{i \Delta_{\ell \ell} L}$ oscillate very fast, and therefore vanish once the finite energy resolution of the detector is taken into account. In the above expression $P_{\alpha \beta}^{(3)}$ is the effective probability derived from the Hamiltonian $H^{(3)}$, which has an ordinary three-neutrino term $H_{\mathrm{SM}}$ (including the usual charged-current interaction term of the electron neutrino) plus a matter term arising from the sterile part of $\tilde{U}^{\dagger} V \tilde{U}$ :

$$
\begin{aligned}
H^{(3)}= & H_{\mathrm{SM}} \\
& \pm \sqrt{2} G_{F} \frac{N_{n}}{2} \sum_{\alpha=\text { sterile }}\left(\begin{array}{ccc}
0 & 0 & 0 \\
0 & \left|\tilde{U}_{\alpha 2}\right|^{2} & \tilde{U}_{\alpha 2}^{*} \tilde{U}_{\alpha 3} \\
0 & \tilde{U}_{\alpha 2} \tilde{U}_{\alpha 3}^{*} & \left|\tilde{U}_{\alpha 3}\right|^{2}
\end{array}\right)
\end{aligned}
$$

Equations (B10) and (B11) are valid for any number of extra sterile states.

\section{APPENDIX C: ROBUSTNESS OF THE ATM + LBL BOUND ON $\boldsymbol{d}_{\boldsymbol{\mu}}$}

As discussed in Refs. [25,28], the contribution of atmospheric neutrino data to the disappearance data set plays a crucial role in rejecting sterile neutrino models. In this Appendix we reconsider the bound on $d_{\mu}$ in $(3+1)$ schemes, generalize it to the $(3+s)$ case, and investigate the impact of some of the adopted approximations. 


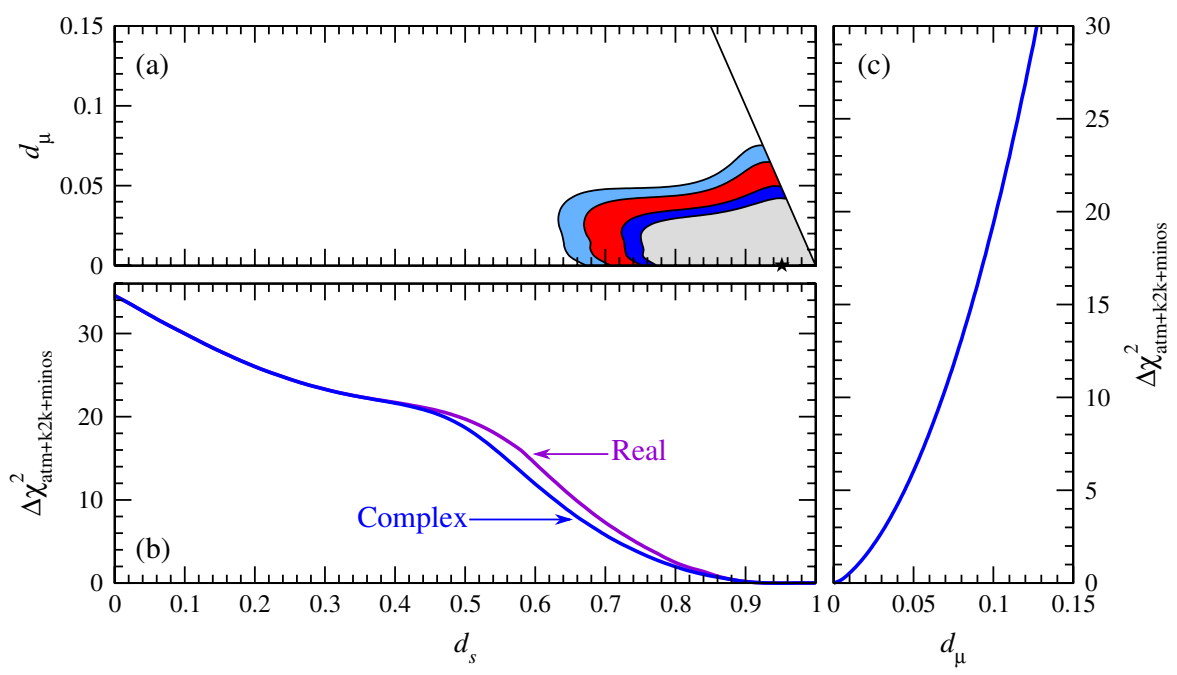

FIG. 11 (color online). Allowed regions for atmospheric and long-baseline data in $(3+1)$ schemes at 90\%, 95\%, 99\%, 99.73\% C.L. ( 2 dof), and projections of $\Delta \chi^{2}$ over $d_{s}=\left|U_{s 4}\right|^{2}$ and $d_{\mu}=\left|U_{\mu 4}\right|^{2}$. In (b) the purple line ("Real") is obtained by forcing the complex phase $\varphi_{34}$ to $0^{\circ}$ or $180^{\circ}$, while for the blue line ("Complex") $\varphi_{34}$ is left free. In (c) the purple line is hidden by the blue one.

\section{Decoupling electron neutrinos}

Let us begin by considering the simplified case $\Delta m_{21}^{2}=0$ and $\theta_{13}=0$. In this limit, the electron neutrino state is completely decoupled, so that $P_{e e}=1$ and $P_{e \mu}=P_{\mu e}=0 .{ }^{4}$ Equations (B10) and (B11) then reduce to an effective two-neutrino form in the $\mu-\tau$ sector:

$$
\begin{gathered}
P_{\mu \mu}=\left(1-d_{\mu}\right)^{2} P_{\mu \mu}^{(2)}+\sum_{\ell \geq 4}\left|U_{\mu \ell}\right|^{4}, \\
H^{(2)}=\frac{\Delta m_{31}^{2}}{4 E}\left(\begin{array}{cc}
-\cos 2 \theta_{23} & \sin 2 \theta_{23} \\
\sin 2 \theta_{23} & \cos 2 \theta_{23}
\end{array}\right) \\
\pm \sqrt{2} G_{F} \frac{N_{n}}{2} \sum_{\alpha=\text { sterile }}\left(\begin{array}{cc}
\left|\tilde{U}_{\alpha 2}\right|^{2} & \tilde{U}_{\alpha 2}^{*} \tilde{U}_{\alpha 3} \\
\tilde{U}_{\alpha 2} \tilde{U}_{\alpha 3}^{*} & \left|\tilde{U}_{\alpha 3}\right|^{2}
\end{array}\right) .
\end{gathered}
$$

In the case of only one extra sterile state it is possible to perform a full numerical analysis. The details of such an analysis have been widely discussed in Refs. [19,21,49], and are summarized in Fig. 11. As can be seen from this figure, atmospheric and long-baseline neutrino data strongly prefer a pure two-neutrino $\nu_{\mu} \rightarrow \nu_{\tau}$ oscillation scenario, disfavoring both a sterile neutrino contribution to the main $\Delta m_{\mathrm{atm}}^{2}$ oscillations (parametrized by $d_{s}=\left|U_{s 4}\right|^{2}$ ) and a mixing of $\nu_{\mu}$ with the heavy mass eigenstate (parametrized by $d_{\mu}=\left|U_{\mu 4}\right|^{2}$ ). In this work we are mainly interested in the bound on $d_{\mu}$, since the other parameters are private to atmospheric and long-baseline data and can therefore be marginalized.

Let us now turn our attention to five-neutrino models. Even in this case it is possible to perform a full numerical

\footnotetext{
${ }^{4} \mathrm{Up}$ to now this approximation has been always adopted in the literature, and in Appendix $\mathrm{C} 2$ we are going to relax it for the first time.
}

analysis, presented in Fig. 12(a). As mentioned in Appendix B, in principle atmospheric and long-baseline data constrain separately $\left|U_{\mu 4}\right|^{2}$ and $\left|U_{\mu 5}\right|^{2}$, which we parametrize in terms of $d_{\mu}$ and $\xi_{\mu} \equiv\left(\left|U_{\mu 4}\right|^{2}-\right.$ $\left.\left|U_{\mu 5}\right|^{2}\right) / d_{\mu}$. However, as can be seen from Fig. 12(a) the allowed region is practically independent of $\xi_{\mu}$. Furthermore, comparing this figure with Fig. 11(c) it turns out that the bound on $d_{\mu}$ in four-neutrino and five-neutrino models is practically the same. In other words, the extra freedom which we have in $5 \nu$ schemes with respect to $4 \nu$ ones is essentially irrelevant for the constraint on $d_{\mu}$.

In order to understand this result, let us go back to Eqs. (C1) and (C2) and consider the differences between $4 \nu$ and $5 \nu$ models. They arise from two facts:

(i) In $4 \nu$ models we have $P_{\mu \mu}=\left(1-d_{\mu}\right)^{2} P_{\mu \mu}^{(2)}+d_{\mu}^{2}$, so that the "scaling" term $\left(1-d_{\mu}\right)^{2}$ and the "constant" term $\sum_{\ell \geq 4}\left|U_{\mu \ell}\right|^{4}$ in Eq. (C1) are related to each other. Conversely, in $5 \nu$ models $P_{\mu \mu}=(1-$ $\left.d_{\mu}\right)^{2} P_{\mu \mu}^{(2)}+d_{\mu}^{2}\left(1+\xi_{\mu}^{2}\right) / 2$, so that the two terms are independent.

(ii) The expression for $P_{\mu \mu}^{(2)}$ is different in the two models, due to the different contributions to the sterile matter term in Eq. (C2). Again, in fiveneutrino models we have more freedom.

The relevance of these differences is illustrated in Fig. 12(b). All the lines correspond to four-neutrino models. The blue line (labeled "Exact") is the exact bound on $d_{\mu}$ from atmospheric and long-baseline data, and coincides with the one shown in Fig. 11(c). The red line (labeled "Vacuum") is obtained by neglecting the last term in Eq. (C2), i.e., by considering only the vacuum part of $H^{(2)}$. Finally, the green line (labeled "Scaling") is obtained 

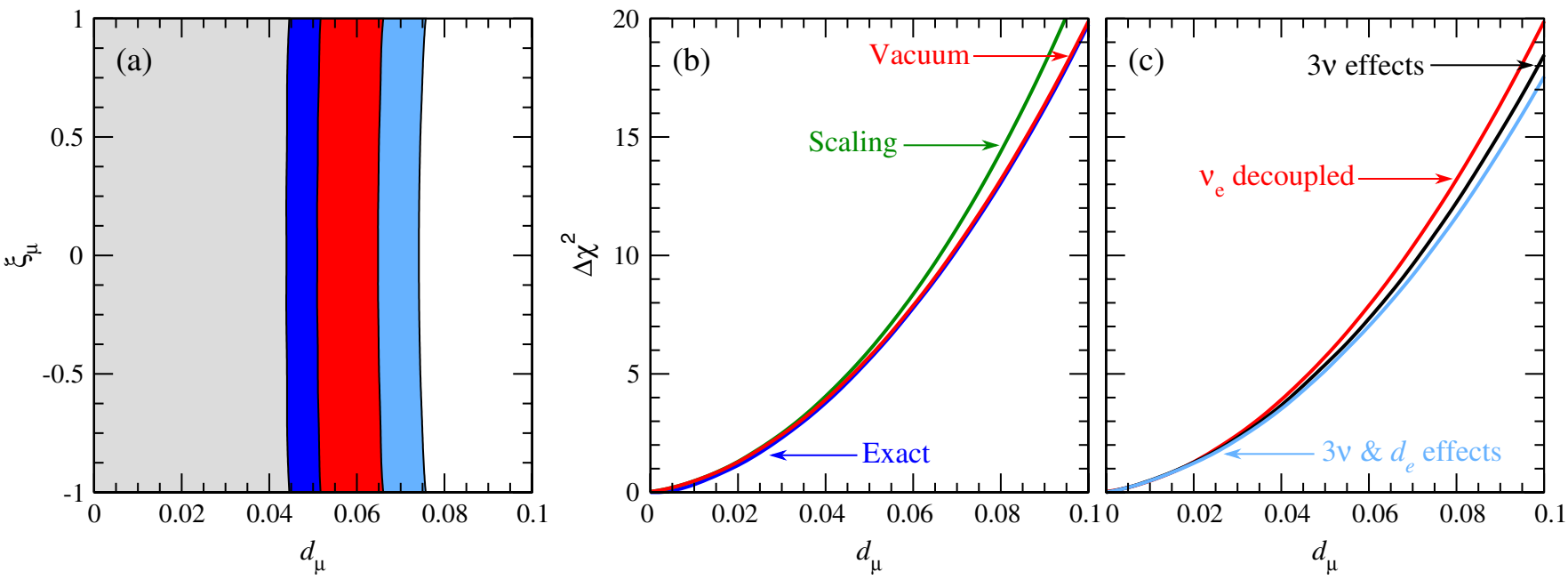

FIG. 12 (color online). (a) Allowed regions at 90\%, 95\%, 99\%, 99.73\% C.L. (2 dof) in the $\left(d_{\mu}, \xi_{\mu}\right)$ plane from the analysis of ATM + LBL data in $(3+2)$ schemes. (b),(c) Impact of different approximations on the atmospheric and long-baseline bound on $d_{\mu}$; see text for details.

by further neglecting the constant term in Eq. (C1), thus setting $P_{\mu \mu}=\left(1-d_{\mu}\right)^{2} P_{\mu \mu}^{(2)}$. As can be seen, sterileinduced matter terms are completely irrelevant for what concerns the bound on $d_{\mu}$, and the constant term in the expression for $P_{\mu \mu}$ plays only a minor role. The real bound arises from the scaling term in $P_{\mu \mu}$, which is the same in four-neutrino and five-neutrino models. This explains why the differences between the two schemes are so small. Similarly, the weak dependence on $\xi_{\mu}$ in $5 \nu$ models arises from the constant term in Eq. (C1); in particular, both Figs. 12(a) and 12(b) show that as this term is decreased the quality of the fit gets worse.

In summary, although in principle atmospheric and longbaseline data could constrain separately all the $\left|U_{\mu \ell}\right|^{2}$ terms in models with extra sterile states, in practice they are only sensitive to the sum of these terms, $d_{\mu}=$ $\sum_{\ell \geq 4}\left|U_{\mu \ell}\right|^{2}$. Furthermore, the bound on $d_{\mu}$ is essentially independent on the number of extra sterile states. The validity of this approximation is crucial for the analysis of $(3+3)$ models, where an exact treatment would be very hard to do due to the large number of parameters involved.

\section{Including electron neutrinos}

In the previous section we have seen that the bound on $d_{\mu}$ reflects the ability of atmospheric and long-baseline data to effectively fix the total normalization of $\mu$-like events. This is possible in spite of the large normalization errors (of the order of 20\%) on the atmospheric neutrino fluxes and the neutrino-nucleon cross sections, since the accurate measurements of $e$-like neutrino events provided by atmospheric data allows one to effectively resolve these uncertainties. In other words, what really matters to determine the bound on $d_{\mu}$ is the relative normalization of $\mu$-like and $e$-like neutrino events. This opens up the pos- sibility that subleading effects modifying the distribution of electron neutrino data may have a sizable impact on the bound on $d_{\mu}$. In this section we investigate in detail this possibility.

In the context of sterile neutrino schemes there are two types of contributions which affect $e$-like events: (i) "usual" three-neutrino effects induced by $\Delta m_{21}^{2}$ or by $\theta_{13}$, and (ii) genuine sterile- $\nu$ effects induced by nonzero $\theta_{1 \ell}$ (with $\ell \geq 4$ ). The formalism to study the threeneutrino effects has been developed in Appendix B. Following the results of Appendix C 1, we assume that the sterile-induced matter effects in Eq. (B11) can be neglected, in which case the effective Hamiltonian $H^{(3)}$ and the corresponding probabilities $P_{\alpha \beta}^{(3)}$ reduce to the usual three-neutrino expressions. Note that again the relevant probabilities are related to the three-neutrino ones only through the parameter $d_{\mu}$, and apart from the constant term in the expression for $P_{\mu \mu}$ in Eq. (B10) (see previous section) these formulas are completely independent from the number of extra sterile species. The results of our analysis are summarized in Fig. 12(c), where we compare the $d_{\mu}$ bound for the case when the electron is decoupled (red line " $v_{e}$ decoupled") with the same bound including also three-neutrino effects due to $\theta_{13}$ and $\Delta m_{21}^{2}$ (black line). Note that the Chooz experiment is also included in the fit. As can be seen from this figure, an accurate treatment of subleading three-neutrino effects indeed weakens the bound on $d_{\mu}$, however the effect is very small and has no impact on the conclusions of this work.

Let us now study effects induced by nonzero values of $\theta_{1 \ell}$. Following the derivation of Appendix B, it is easy to see that in this case $\tilde{U}_{\mu 1}$ and $\tilde{U}_{\mu 3}$ no longer vanish, in which case Eq. (B10) should be replaced with expressions involving not only $P_{\alpha \beta}^{(3)}$ but also interference terms between 
different entries of $\tilde{S}$. However, it is still true that $\tilde{U}_{e 2}=$ $\tilde{U}_{e 3}=0$, so that

$P_{e e}=\left(1-d_{e}\right)^{2} P_{e e}^{(3)}+\sum_{\ell \geq 4}\left|U_{e \ell}\right|^{4}, \quad$ with $\quad d_{e} \equiv \sum_{\ell \geq 4}\left|U_{e \ell}\right|^{2}$.

Motivated by this result, we approximate the effects of nonzero $\theta_{1 \ell}$ by introducing an independent scaling factor for electron events:

$$
N_{e}\left(d_{e}\right)=\left(1-2 d_{e}\right) N_{e}(0) .
$$

If $d_{e}$ is left free to vary without any constraint, the impact on the bound on $d_{\mu}$ is dramatic, since in this case electron events can no longer fix the $20 \%$ flux and cross section normalization uncertainties. However, the value of $d_{e}$ is strongly bounded by Bugey. We have performed a combined analysis of atmospheric + LBL and SBL data taking into account that both data samples depend on $d_{\mu}$ and $d_{e}$ (using the approximation described above). We find that the final result is practically the same as in our standard case, where the dependence of the ATM data on $d_{e}$ is neglected. To illustrate this we show in Fig. 12(c), light blue line (" $3 v \& d_{e}$ effects"), also the bound on $d_{\mu}$ from ATM data by adding to the overall $\chi^{2}$ a term $\left(d_{e} / 0.012\right)^{2}$, which simulates roughly the constraint from Bugey, neglecting that it actually depends on $\Delta m_{41}^{2}$. From this figure it becomes clear that once $d_{e}$ is limited by the data from Bugey its impact on atmospheric + LBL data is very small.

In conclusion, the atmospheric bound on $d_{\mu}$ is robust under our approximations. However, as clear from the above discussion it depends on the assumptions about uncertainties on quantities (like fluxes or cross sections) affecting the ratio of $e$-like and $\mu$-like atmospheric neutrino event normalizations.
[1] A. A. Aguilar-Arevalo et al. (MiniBooNE Collaboration), Phys. Rev. Lett. 98, 231801 (2007).

[2] W. Louis and J. Conrad (MiniBooNE Collaboration), http://www-boone.fnal.gov/publicpages/First_Results.pdf.

[3] A. Aguilar et al. (LSND Collaboration), Phys. Rev. D 64, 112007 (2001).

[4] Y. Fukuda et al. (Super-Kamiokande Collaboration), Phys. Rev. Lett. 81, 1562 (1998); Y. Ashie et al., Phys. Rev. D 71, 112005 (2005).

[5] E. Aliu et al. (K2K Collaboration), Phys. Rev. Lett. 94, 081802 (2005).

[6] D. G. Michael et al. (MINOS Collaboration), Phys. Rev. Lett. 97, 191801 (2006).

[7] B. T. Cleveland et al., Astrophys. J. 496, 505 (1998); J. N. Abdurashitov et al. (SAGE Collaboration), J. Exp. Theor. Phys. 95, 181 (2002); M. Altmann et al. (GNO Collaboration), Phys. Lett. B 616, 174 (2005).

[8] S. Fukuda et al. (Super-Kamkiokande Collaboration), Phys. Lett. B 539, 179 (2002); J. Hosaka et al., Phys. Rev. D 73, 112001 (2006).

[9] Q. R. Ahmad et al. (SNO Collaboration), Phys. Rev. Lett. 89, 011301 (2002); B. Aharmim et al., Phys. Rev. C 72, 055502 (2005).

[10] K. Eguchi et al. (KamLAND Collaboration), Phys. Rev. Lett. 90, 021802 (2003); T. Araki et al., Phys. Rev. Lett. 94, 081801 (2005).

[11] J. T. Peltoniemi, D. Tommasini, and J. W. F. Valle, Phys. Lett. B 298, 383 (1993); J. T. Peltoniemi and J. W. F. Valle, Nucl. Phys. B406, 409 (1993); D. O. Caldwell and R. N. Mohapatra, Phys. Rev. D 48, 3259 (1993).

[12] M. Maltoni, T. Schwetz, M. A. Tortola, and J. W. F. Valle, Nucl. Phys. B643, 321 (2002).

[13] A. Strumia, Phys. Lett. B 539, 91 (2002).
[14] B. Armbruster et al. (KARMEN Collaboration), Phys. Rev. D 65, 112001 (2002).

[15] P. Astier et al. (NOMAD Collaboration), Phys. Lett. B 570, 19 (2003).

[16] F. Dydak et al., Phys. Lett. B 134, 281 (1984).

[17] Y. Declais et al., Nucl. Phys. B434, 503 (1995).

[18] J. J. Gomez-Cadenas and M. C. Gonzalez-Garcia, Z. Phys. C 71, 443 (1996); S. Goswami, Phys. Rev. D 55, 2931 (1997).

[19] M. Maltoni, T. Schwetz, M. A. Tortola, and J. W. F. Valle, New J. Phys. 6, 122 (2004).

[20] M. C. Gonzalez-Garcia and M. Maltoni, arXiv:0704.1800.

[21] C. Giunti, M.C. Gonzalez-Garcia, and C. Pena-Garay, Phys. Rev. D 62, 013005 (2000); M. C. GonzalezGarcia, M. Maltoni, and C. Pena-Garay, Phys. Rev. D 64, 093001 (2001); M. Maltoni, T. Schwetz, M. A. Tortola, and J.W.F. Valle, Phys. Rev. D 67, 013011 (2003).

[22] S. M. Bilenky, C. Giunti, and W. Grimus, Eur. Phys. J. C 1, 247 (1998).

[23] N. Okada and O. Yasuda, Int. J. Mod. Phys. A 12, 3669 (1997).

[24] V. D. Barger, S. Pakvasa, T. J. Weiler, and K. Whisnant, Phys. Rev. D 58, 093016 (1998).

[25] S. M. Bilenky, C. Giunti, W. Grimus, and T. Schwetz, Phys. Rev. D 60, 073007 (1999).

[26] O. L. G. Peres and A. Y. Smirnov, Nucl. Phys. B599, 3 (2001).

[27] W. Grimus and T. Schwetz, Eur. Phys. J. C 20, 1 (2001).

[28] M. Maltoni, T. Schwetz, and J. W. F. Valle, Phys. Lett. B 518, 252 (2001).

[29] M. Sorel, J. M. Conrad, and M. Shaevitz, Phys. Rev. D 70, 073004 (2004). 
[30] E. Ma, G. Rajasekaran, and I. Stancu, Phys. Rev. D 61, 071302 (2000); E. Ma and G. Rajasekaran, Phys. Rev. D 64, 117303 (2001).

[31] S. Palomares-Ruiz, S. Pascoli, and T. Schwetz, J. High Energy Phys. 09 (2005) 048

[32] H. Murayama and T. Yanagida, Phys. Lett. B 520, 263 (2001); G. Barenboim, L. Borissov, and J. Lykken, arXiv:hep-ph/0212116; M. C. Gonzalez-Garcia, M. Maltoni, and T. Schwetz, Phys. Rev. D 68, 053007 (2003); V. Barger, D. Marfatia, and K. Whisnant, Phys. Lett. B 576, 303 (2003).

[33] V. A. Kostelecky and M. Mewes, Phys. Rev. D 70, 076002 (2004); A. de Gouvea and Y. Grossman, Phys. Rev. D 74, 093008 (2006); T. Katori, A. Kostelecky, and R. Tayloe, Phys. Rev. D 74, 105009 (2006).

[34] K.S. Babu and S. Pakvasa, arXiv:hep-ph/0204236; B. Armbruster et al. (KARMEN Collaboration), Phys. Rev. Lett. 90, 181804 (2003); A. Gaponenko et al. (TWIST Collaboration), Phys. Rev. D 71, 071101 (2005).

[35] G. Barenboim and N.E. Mavromatos, J. High Energy Phys. 01 (2005) 034.

[36] D. B. Kaplan, A.E. Nelson, and N. Weiner, Phys. Rev. Lett. 93, 091801 (2004); K. M. Zurek, J. High Energy Phys. 10 (2004) 058; V. Barger, D. Marfatia, and K. Whisnant, Phys. Rev. D 73, 013005 (2006).

[37] H. Pas, S. Pakvasa, and T.J. Weiler, Phys. Rev. D 72, 095017 (2005).

[38] M. Cirelli, G. Marandella, A. Strumia, and F. Vissani, Nucl. Phys. B708, 215 (2005).

[39] K. Enqvist, K. Kainulainen, and M. J. Thomson, Nucl. Phys. B373, 498 (1992); X. Shi, D. N. Schramm, and B. D. Fields, Phys. Rev. D 48, 2563 (1993); S. M. Bilenky, C. Giunti, W. Grimus, and T. Schwetz, Astropart. Phys. 11, 413 (1999); P. Di Bari, Phys. Rev. D 65, 043509
(2002); 67, 127301 (2003).

[40] S. Hannestad and G. G. Raffelt, J. Cosmol. Astropart. Phys. 11 (2006) 016; S. Dodelson, A. Melchiorri, and A. Slosar, Phys. Rev. Lett. 97, 041301 (2006).

[41] R. Foot and R. R. Volkas, Phys. Rev. Lett. 75, 4350 (1995); Y.Z. Chu and M. Cirelli, Phys. Rev. D 74, 085015 (2006); C. J. Smith, G. M. Fuller, C. T. Kishimoto, and K. N. Abazajian, Phys. Rev. D 74, 085008 (2006).

[42] K. S. Babu and I.Z. Rothstein, Phys. Lett. B 275, 112 (1992); L. Bento and Z. Berezhiani, Phys. Rev. D 64, 115015 (2001).

[43] G. Gelmini, S. Palomares-Ruiz, and S. Pascoli, Phys. Rev. Lett. 93, 081302 (2004).

[44] E. D. Church, K. Eitel, G. B. Mills, and M. Steidl, Phys. Rev. D 66, 013001 (2002).

[45] Technical data on the MiniBooNE oscillation analysis is available at http://www-boone.fnal.gov/for_physicists/ april07datarelease/.

[46] M. Maltoni and T. Schwetz, Phys. Rev. D 68, 033020 (2003).

[47] M. Apollonio et al., Eur. Phys. J. C 27, 331 (2003).

[48] F. Boehm et al., Phys. Rev. D 64, 112001 (2001).

[49] M. Maltoni, T. Schwetz, and J. W. F. Valle, Phys. Rev. D 65, 093004 (2002).

[50] G. Karagiorgi et al., Phys. Rev. D 75, 013011 (2007).

[51] F. Ardellier et al. (Double Chooz Collaboration), arXiv:hep-ex/0606025; X. Guo et al. (Daya Bay Collaboration), arXiv:hep-ex/0701029.

[52] C. Giunti and M. Laveder, arXiv:hep-ph/0610352; C. Grieb, J. Link, and R.S. Raghavan, Phys. Rev. D 75, 093006 (2007).

[53] Y. Itow et al., arXiv:hep-ex/0106019; D. S. Ayres et al. (NOvA Collaboration), arXiv:hep-ex/0503053.

[54] G. T. Garvey et al., Phys. Rev. D 72, 092001 (2005). 